\title{
GEODESIC COMPLETENESS FOR SOBOLEV METRICS ON THE SPACE OF IMMERSED PLANE CURVES
}

\author{
MARTINS BRUVERIS ${ }^{1}$, PETER W. MICHOR ${ }^{2}$ and DAVID MUMFORD ${ }^{3}$ \\ ${ }^{1}$ Institut de Mathématiques, EPFL, Lausanne 1015, Switzerland; \\ email: martins.bruveris@epfl.ch \\ ${ }^{2}$ Fakultät für Mathematik, Universität Wien, Oskar-Morgenstern-Platz 1, Wien 1090, Austria \\ ${ }^{3}$ Division of Applied Mathematics, Brown University, Box F, Providence, RI 02912, USA
}

Received 19 December 2013; accepted 3 July 2014

\begin{abstract}
We study properties of Sobolev-type metrics on the space of immersed plane curves. We show that the geodesic equation for Sobolev-type metrics with constant coefficients of order 2 and higher is globally well-posed for smooth initial data as well as for initial data in certain Sobolev spaces. Thus the space of closed plane curves equipped with such a metric is geodesically complete. We find lower bounds for the geodesic distance in terms of curvature and its derivatives.
\end{abstract}

2010 Mathematics Subject Classification: 58D15 (primary); 35G55, 53A04, 58B20 (secondary)

\section{Introduction}

Sobolev-type metrics on the space of plane immersed curves were independently introduced in $[7,17,24]$. They are used in computer vision, shape classification, and tracking, mainly in the form of their induced metric on shape space, which is the orbit space under the action of the reparameterization group. See $[14,23]$ for applications of Sobolev-type metrics and $[2,18]$ for an overview of their mathematical properties. Sobolev-type metrics were also generalized to immersions of higher-dimensional manifolds in $[4,5]$.

It was shown in [18] that the geodesic equation of a Sobolev-type metric of order $n \geqslant 1$ is locally well-posed, and this result was extended in [4] to a larger class of metrics and immersions of arbitrary dimension. The main result of this 
paper is to show global well-posedness of the geodesic equation for Sobolev-type metrics of order $n \geqslant 2$ with constant coefficients. In particular, we prove the following theorem.

THEOREM 1.1. Let $n \geqslant 2$, and let the metric $G$ on $\operatorname{Imm}\left(S^{1}, \mathbb{R}^{2}\right)$ be given by

$$
G_{c}(h, k)=\int_{S^{1}} \sum_{j=0}^{n} a_{j}\left\langle D_{s}^{j} h, D_{s}^{j} k\right\rangle \mathrm{d} s,
$$

with $a_{j} \geqslant 0$ and $a_{0}, a_{n} \neq 0$. Given initial conditions $\left(c_{0}, u_{0}\right) \in T \operatorname{Imm}\left(S^{1}, \mathbb{R}^{2}\right)$, the solution of the geodesic equation

$$
\begin{aligned}
\partial_{t}\left(\sum_{j=0}^{n}(-1)^{j} a_{j}\left|c^{\prime}\right| D_{s}^{2 j} c_{t}\right)= & -\frac{a_{0}}{2}\left|c^{\prime}\right| D_{s}\left(\left\langle c_{t}, c_{t}\right\rangle v\right) \\
& +\sum_{k=1}^{n} \sum_{j=1}^{2 k-1}(-1)^{k+j} \frac{a_{k}}{2}\left|c^{\prime}\right| D_{s}\left(\left\langle D_{s}^{2 k-j} c_{t}, D_{s}^{j} c_{t}\right\rangle v\right)
\end{aligned}
$$

for the metric $G$ with initial values $\left(c_{0}, u_{0}\right)$ exists for all time.

Here, $\operatorname{Imm}\left(S^{1}, \mathbb{R}^{2}\right)$ denotes the space of all smooth, closed, plane curves with nowhere zero tangent vectors; this space is open in $C^{\infty}\left(S^{1}, \mathbb{R}^{2}\right)$. We assume that $c \in \operatorname{Imm}\left(S^{1}, \mathbb{R}^{2}\right)$ and $h, k$ are vector fields along $c, \mathrm{~d} s=\left|c^{\prime}\right| \mathrm{d} \theta$ is the arc length measure, $D_{s}=\left(1 /\left|c^{\prime}\right|\right) \partial_{\theta}$ is the derivative with respect to arc length, $v=c^{\prime} /\left|c^{\prime}\right|$ is the unit length tangent vector to $c$, and $\langle\cdot, \cdot\rangle$ is the Euclidean inner product on $\mathbb{R}^{2}$.

Thus, if $G$ is a Sobolev-type metric of order at least 2, then the Riemannian manifold $\left(\operatorname{Imm}\left(S^{1}, \mathbb{R}^{2}\right), G\right)$ is geodesically complete. If the Sobolev-type metric is invariant under the reparameterization group $\operatorname{Diff}\left(S^{1}\right)$, the induced metric on shape space $\operatorname{Imm}\left(S^{1}, \mathbb{R}^{2}\right) / \operatorname{Diff}\left(S^{1}\right)$ is also geodesically complete. The latter space is an infinite-dimensional orbifold; see [17, 2.5 and 2.10].

Theorem 1.1 seems to be the first result about geodesic completeness on manifolds of mappings outside the realm of diffeomorphism groups and manifolds of metrics. In the first paragraph of [9, p. 140], a proof is sketched that a right invariant $H^{s}$-metric on the group of volume-preserving diffeomorphisms on a compact manifold $M$ is geodesically complete if $s>\operatorname{dim}(M) / 2+1$. In [25], there is an implicit result that a group of diffeomorphisms constructed from a reproducing kernel Hilbert space of vector fields whose reproducing kernel is at least $C^{1}$ is geodesically complete. For a certain metric on a group of diffeomorphisms on $\mathbb{R}^{n}$ with $C^{1}$ kernel, geodesic completeness is shown in [19, Theorem 2]. Metric completeness and existence of minimizing geodesics 
have also been studied on the diffeomorphism group in [6]. The manifold of all Riemannian metrics with fixed volume form is geodesically complete for the $L^{2}$-metric (also called the Ebin metric).

Sobolev-type metrics of order 1 are not geodesically complete, since it is possible to shrink a circle to a point along a geodesic in finite time; see [18, Section 6.1]. Similarly, a Sobolev metric of order 2 or higher with both $a_{0}$, $a_{1}=0$ is a geodesically incomplete metric on the space $\operatorname{Imm}\left(S^{1}, \mathbb{R}^{2}\right) / \operatorname{Tra}$ of plane curves modulo translations. In this case, it is possible to blow up a circle along a geodesic to infinity in finite time; see Remark 5.7.

In order to prove long-time existence of geodesics, we need to study properties of the geodesic distance. In particular, we show the following theorem regarding continuity of curvature $\kappa$ and its derivatives.

THEOREM 1.2. Let $G$ be a Sobolev-type metric of order $n \geqslant 2$ with constant coefficients, and let $\mathrm{dist}^{G}$ be the induced geodesic distance. If $0 \leqslant k \leqslant n-2$, then the functions

$$
\begin{array}{r}
D_{s}^{k}(\kappa) \sqrt{\left|c^{\prime}\right|}:\left(\operatorname{Imm}\left(S^{1}, \mathbb{R}^{2}\right), \operatorname{dist}^{G}\right) \rightarrow L^{2}\left(S^{1}, \mathbb{R}\right) \\
D_{s}^{k+1}\left(\log \left|c^{\prime}\right|\right) \sqrt{\left|c^{\prime}\right|}:\left(\operatorname{Imm}\left(S^{1}, \mathbb{R}^{2}\right), \operatorname{dist}^{G}\right) \rightarrow L^{2}\left(S^{1}, \mathbb{R}\right)
\end{array}
$$

are continuous and Lipschitz continuous on every metric ball.

A similar statement can be derived for the $L^{\infty}$-continuity of curvature and its derivatives; see Remark 4.9.

The full proof of Theorem 1.1 is surprisingly complicated. One reason is that we have to work on the Sobolev completion (always with respect to the original parameter $\theta$ in $S^{1}$ ) of the space of immersions in order to apply results on ordinary differential equations (ODEs) on Banach spaces. Here, the operators (and their inverses and adjoints) acquire nonsmooth coefficients. Since we we want the Sobolev order to be as low as possible, the geodesic equation involves $H^{-n}$; see Section 3.3. Eventually, we use that the metric operator has constant coefficients. We have to use estimates with precise constants which are uniformly bounded on metric balls.

In [4], the authors studied Sobolev metrics on immersions of higherdimensional manifolds. One might hope that similar methods to those used in this article can be applied to show the geodesic completeness of the spaces $\operatorname{Imm}(M, N)$ with $M$ compact and $(N, \bar{g})$ a suitable Riemannian manifold. Crucial ingredients in the proof for plane curves are the Sobolev inequalities in Lemmas 2.14 and 2.15 with explicit constants, which only depend on the curve through the length. The lack of such inequalities for general $M$ will one of the factors complicating life in higher dimensions. 


\section{Background material and notation}

\subsection{The space of curves. The space}

$$
\operatorname{Imm}\left(S^{1}, \mathbb{R}^{2}\right)=\left\{c \in C^{\infty}\left(S^{1}, \mathbb{R}^{2}\right): c^{\prime}(\theta) \neq 0\right\}
$$

of immersions is an open set in the Fréchet space $C^{\infty}\left(S^{1}, \mathbb{R}^{2}\right)$ with respect to the $C^{\infty}$-topology, and thus itself is a smooth Fréchet manifold. The tangent space of $\operatorname{Imm}\left(S^{1}, \mathbb{R}^{2}\right)$ at the point $c$ consists of all vector fields along the curve $c$. It can be described as the space of sections of the pullback bundle $c^{*} T \mathbb{R}^{2}$,

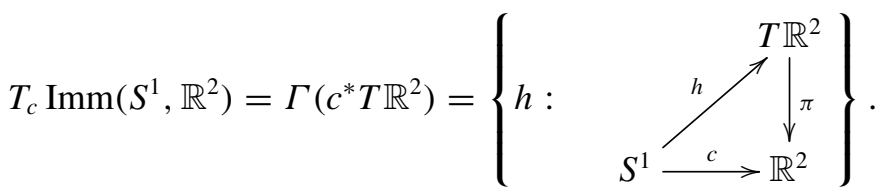

In our case, since the tangent bundle $T \mathbb{R}^{2}$ is trivial, it can also be identified with the space of $\mathbb{R}^{2}$-valued functions on $S^{1}$,

$$
T_{c} \operatorname{Imm}\left(S^{1}, \mathbb{R}^{2}\right) \cong C^{\infty}\left(S^{1}, \mathbb{R}^{2}\right) .
$$

For a curve $c \in \operatorname{Imm}\left(S^{1}, \mathbb{R}^{2}\right)$, we denote the parameter by $\theta \in S^{1}$ and differentiation $\partial_{\theta}$ by ', i.e., $c^{\prime}=\partial_{\theta} c$. Since $c$ is an immersion, the unit-length tangent vector $v=c^{\prime} /\left|c^{\prime}\right|$ is well defined. Rotating $v$ by $\pi / 2$, we obtain the unitlength normal vector $n=J v$, where $J$ is rotation by $\pi / 2$. We will denote by $D_{s}=\partial_{\theta} /\left|c_{\theta}\right|$ the derivative with respect to arc length and by $\mathrm{d} s=\left|c_{\theta}\right| \mathrm{d} \theta$ the integration with respect to arc length. To summarize, we have

$$
v=D_{s} c, \quad n=J v, \quad D_{s}=\frac{1}{\left|c_{\theta}\right|} \partial_{\theta}, \quad \mathrm{d} s=\left|c_{\theta}\right| \mathrm{d} \theta .
$$

The curvature can be defined as

$$
\kappa=\left\langle D_{s} v, n\right\rangle,
$$

and we have the Frenet equations

$$
\begin{aligned}
& D_{s} v=\kappa n \\
& D_{s} n=-\kappa v .
\end{aligned}
$$

The length of a curve will be denoted by $\ell_{c}=\int_{S^{1}} 1 \mathrm{~d} s$. We define the turning angle $\alpha: S^{1} \rightarrow \mathbb{R} / 2 \pi \mathbb{Z}$ of a curve $c$ by $v(\theta)=(\cos \alpha(\theta), \sin \alpha(\theta))$. Then curvature is given by $\kappa=D_{s} \alpha$. 
2.2. Variational formulas. We will need formulas that express how the quantities $v, n$, and $\kappa$ change if we vary the underlying curve $c$. For a smooth map $F$ from $\operatorname{Imm}\left(S^{1}, \mathbb{R}^{2}\right)$ to any convenient vector space (see [13]), we denote by

$$
D_{c, h} F=\left.\frac{\mathrm{d}}{\mathrm{d} t}\right|_{t=0} F(c+t h)
$$

the variation in the direction $h$.

The proof of the following formulas can be found, for example, in [18].

$$
\begin{aligned}
D_{c, h} v & =\left\langle D_{s} h, n\right\rangle n \Longrightarrow D_{c, h} \alpha=\left\langle D_{s} h, n\right\rangle \\
D_{c, h} n & =-\left\langle D_{s} h, n\right\rangle v \\
D_{c, h} \kappa & =\left\langle D_{s}^{2} h, n\right\rangle-2 \kappa\left\langle D_{s} h, v\right\rangle \\
D_{c, h}\left(\left|c^{\prime}\right|^{k}\right) & =k\left\langle D_{s} h, v\right\rangle\left|c^{\prime}\right|^{k} .
\end{aligned}
$$

With these basic building blocks, one can use the following lemma to compute the variations of higher derivatives.

LEMMA 2.3. If $F$ is a smooth map $F: \operatorname{Imm}\left(S^{1}, \mathbb{R}^{2}\right) \rightarrow C^{\infty}\left(S^{1}, \mathbb{R}^{d}\right)$, then the variation of the composition $D_{s} \circ F$ is given by

$$
D_{c, h}\left(D_{s} \circ F\right)=D_{s}\left(D_{c, h} F\right)-\left\langle D_{s} h, v\right\rangle D_{s} F(c) .
$$

Proof. The operator $\partial_{\theta}$ is linear, and thus it commutes with the derivative with respect to $c$. Thus we have

$$
\begin{aligned}
D_{c, h}\left(D_{s} \circ F\right) & =D_{c, h}\left(\left|c^{\prime}\right|^{-1} \partial_{\theta} F(c)\right) \\
& =\left|c^{\prime}\right|^{-1} \partial_{\theta}\left(D_{c, h} F\right)+\left(D_{c, h}\left|c^{\prime}\right|^{-1}\right) \partial_{\theta} F(c) \\
& =D_{s}\left(D_{c, h} F\right)-\left\langle D_{s} h, v\right\rangle\left|c^{\prime}\right|^{-1} \partial_{\theta} F(c) \\
& =D_{s}\left(D_{c, h} F\right)-\left\langle D_{s} h, v\right\rangle D_{s} F(c) .
\end{aligned}
$$

2.4. Sobolev norms. In this paper, we will only consider Sobolev spaces of integer order. For $n \geqslant 1$, the $H^{n}(d \theta)$-norm on $C^{\infty}\left(S^{1}, \mathbb{R}^{d}\right)$ is given by

$$
\|u\|_{H^{n}(d \theta)}^{2}=\int_{S^{1}}|u|^{2}+\left|\partial_{\theta}^{n} u\right|^{2} \mathrm{~d} \theta .
$$

Given $c \in \operatorname{Imm}\left(S^{1}, \mathbb{R}^{2}\right)$, we define the $H^{n}(d s)$-norm on $C^{\infty}\left(S^{1}, \mathbb{R}^{d}\right)$ by

$$
\|u\|_{H^{n}(d s)}^{2}=\int_{S^{1}}|u(s)|^{2}+\left|D_{s}^{n} u(s)\right|^{2} \mathrm{~d} s .
$$


Note that in (2) integration and differentiation are performed with respect to the arc length of $c$, while in (1) the parameter $\theta$ is used. In particular, the $H^{n}(d s)$ norm depends on the curve $c$. The norms $H^{n}(d \theta)$ and $H^{n}(d s)$ are equivalent, but the constants do depend on $c$. We prove in Lemma 5.1 that, if $c$ does not vary too much, the constants can be chosen independently of $c$.

The $L^{2}(d \theta)$ - and $L^{2}(d s)$-norms are defined similarly,

$$
\|u\|_{L^{2}(d \theta)}^{2}=\int_{S^{1}}|u|^{2} \mathrm{~d} \theta, \quad\|u\|_{L^{2}(d s)}^{2}=\int_{S^{1}}|u|^{2} \mathrm{~d} s,
$$

and they are related via $\left\|u \sqrt{\left|c^{\prime}\right|}\right\|_{L^{2}(d \theta)}=\|u\|_{L^{2}(d s)}$. Whenever we write $H^{n}\left(S^{1}\right.$, $\left.\mathbb{R}^{d}\right)$ or $L^{2}\left(S^{1}, \mathbb{R}^{d}\right)$, we always endow them with the $H^{n}(d \theta)$ - and $L^{2}(d \theta)$-norms.

For $n \geqslant 2$, we shall denote by

$$
\operatorname{Imm}^{n}\left(S^{1}, \mathbb{R}^{2}\right)=\left\{c: c \in H^{n}\left(S^{1}, \mathbb{R}^{2}\right), c^{\prime}(\theta) \neq 0\right\}
$$

the space of Sobolev immersions of order $n$. Because of the Sobolev embedding theorem, see [1], we have $H^{2}\left(S^{1}, \mathbb{R}^{2}\right) \hookrightarrow C^{1}\left(S^{1}, \mathbb{R}^{2}\right)$, and thus $\operatorname{Imm}^{n}\left(S^{1}, \mathbb{R}^{2}\right)$ is well-defined. We will see in Section 3.2 that the $H^{n}(d s)$-norm remains welldefined if $c \in \operatorname{Imm}^{n}\left(S^{1}, \mathbb{R}^{2}\right)$.

The following result on pointwise multiplication will be used repeatedly. It can be found, among other places, in [11, Lemma 2.3]. We will in particular use that $k$ can be negative.

LEMMA 2.5. Let $n \geqslant 1$, and let $k \in \mathbb{Z}$ with $|k| \leqslant n$. Then pointwise multiplication induces a bounded bilinear map.

$$
\cdot: H^{n}\left(S^{1}, \mathbb{R}^{d}\right) \times H^{k}\left(S^{1}, \mathbb{R}^{d}\right) \rightarrow H^{k}\left(S^{1}, \mathbb{R}\right), \quad(f, g) \mapsto\langle f, g\rangle .
$$

The last tool that we will need is composition of Sobolev diffeomorphisms. For $n \geqslant 1$, define

$$
\mathcal{D}^{n}\left(S^{1}\right)=\left\{\varphi: \varphi \text { is } C^{1} \text {-diffeomorphism of } S^{1} \text { and } \varphi \in H^{n}\left(S^{1}, S^{1}\right)\right\}
$$

to be the group of Sobolev diffeomorphisms. The following lemma can be found in [11, Theorem 1.2].

LEMMA 2.6. Let $n \geqslant 2$, and let $0 \leqslant k \leqslant n$. Then the composition map

$$
H^{k}\left(S^{1}, \mathbb{R}^{d}\right) \times \mathcal{D}^{n}\left(S^{1}\right) \rightarrow H^{k}\left(S^{1}, \mathbb{R}^{d}\right), \quad(f, \varphi) \mapsto f \circ \varphi
$$

is continuous. 
Let $n \geqslant 2$, and fix $\varphi \in \mathcal{D}^{n}\left(S^{1}\right)$. Denote by $R_{\varphi}(h)=h \circ \varphi$ the composition with $\varphi$. From Lemma 2.6, we see that $R_{\varphi}$ is a bounded linear map $R_{\varphi}: H^{n} \rightarrow H^{n}$. The following lemma tells us that the transpose of this map respects Sobolev orders.

LEMMA 2.7. Let $n \geqslant 2, \varphi \in \mathcal{D}^{n}\left(S^{1}\right)$, and $-n \leqslant k \leqslant n-1$. Then the restrictions of $R_{\varphi}^{*}$ are bounded linear maps

$$
R_{\varphi}^{*} \uparrow H^{k}\left(S^{1}, \mathbb{R}^{d}\right): H^{k}\left(S^{1}, \mathbb{R}^{d}\right) \rightarrow H^{k}\left(S^{1}, \mathbb{R}^{d}\right) .
$$

On $L^{2}\left(S^{1}, \mathbb{R}^{d}\right)$, we have the identity $R_{\varphi^{-1}}^{*}(f)=R_{\varphi}(f) \varphi^{\prime}$.

Proof. For $-n \leqslant k \leqslant 0$, we obtain from Lemma 2.6 that $R_{\varphi}$ is a map $R_{\varphi}: H^{-k} \rightarrow$ $H^{-k}$, and by $L^{2}$-duality we obtain that $R_{\varphi}^{*}: H^{k} \rightarrow H^{k}$, as required.

Now let $0 \leqslant k \leqslant n-1, f \in H^{k}$, and $g \in H^{n}$. We replace $\varphi$ by $\varphi^{-1}$ to simplify the formulas. By definition of the transpose,

$$
\begin{aligned}
\left\langle R_{\varphi^{-1}}^{*}\right. & f, g\rangle_{H^{-n} \times H^{n}}=\left\langle f, R_{\varphi^{-1}} g\right\rangle_{H^{-n} \times H^{n}} \\
& =\int_{S^{1}}\left\langle f(\theta), g\left(\varphi^{-1}(\theta)\right)\right\rangle \mathrm{d} \theta=\int_{S^{1}}\langle f(\varphi(\theta)), g(\theta)\rangle \varphi^{\prime}(\theta) \mathrm{d} \theta \\
& =\left\langle\left(R_{\varphi} f\right) \varphi^{\prime}, g\right\rangle_{H^{-p} \times H^{p}} .
\end{aligned}
$$

Thus we obtain $R_{\varphi^{-1}}^{*}(f)=R_{\varphi}(f) \varphi^{\prime}$, and using Lemma 2.5 we see that for $f \in$ $H^{k}$ we also have $R_{\varphi^{-1}}^{*}(f) \in H^{k}$.

2.8. Notation. We will write

$$
f \lesssim_{A} g
$$

if there exists a constant $C>0$, possibly depending on $A$, such that the inequality $f \leqslant C g$ holds.

2.9. Gronwall inequalities. The following version of Gronwall's inequality can be found in [22, Theorem 1.3.2] and [12].

THEOREM 2.10. Let $A, \Phi$, and $\Psi$ be real continuous functions defined on $[a, b]$, and let $\Phi \geqslant 0$. We suppose that on $[a, b]$ we have the following inequality:

$$
A(t) \leqslant \Psi(t)+\int_{a}^{t} A(s) \Phi(s) \mathrm{d} s .
$$


Then

holds on $[a, b]$.

$$
A(t) \leqslant \Psi(t)+\int_{a}^{t} \Psi(s) \Phi(s) \exp \left(\int_{s}^{t} \Phi(u) \mathrm{d} u\right) \mathrm{d} s
$$

We will repeatedly use the following corollary.

COROLlaRY 2.11. Let $A$ and $G$ be real continuous functions on $[0, T]$ with $G \geqslant$ 0 , and let $\alpha$ and $\beta$ be nonnegative constants. We suppose that on $[0, T]$ we have the inequality

$$
A(t) \leqslant A(0)+\int_{0}^{t}(\alpha+\beta A(s)) G(s) \mathrm{d} s .
$$

Then

$$
A(t) \leqslant A(0)+\left(\alpha+(A(0)+\alpha N) \beta e^{\beta N}\right) \int_{0}^{t} G(s) \mathrm{d} s
$$

holds in $[0, T]$ with $N=\int_{0}^{T} G(t) \mathrm{d} t$.

Proof. Apply the Gronwall inequality with $[a, b]=[0, T], \Psi(t)=$ $A(0)+\alpha \int_{0}^{t} G(s) \mathrm{d} s$ and $\Phi(s)=\beta G(s)$, and note that $G(s) \geqslant 0$ implies $\int_{s}^{t} G(u) \mathrm{d} u \leqslant N$.

2.12. Poincaré inequalities. In the later sections it will be necessary to estimate the $H^{k}(d s)$-norm of a function by the $H^{n}(d s)$-norm with $k<n$, as well as the $L^{\infty}$-norm by the $H^{k}(d s)$-norm. In particular, we will need to know how the curve $c$ enters into the estimates. The basic result is the following lemma, which is adapted from [15, Lemma 18].

LeMma 2.13. Let $c \in \operatorname{Imm}^{2}\left(S^{1}, \mathbb{R}^{2}\right)$ and let $h: S^{1} \rightarrow \mathbb{R}^{d}$ be absolutely continuous. Then

$$
\sup _{\theta \in S^{1}}\left|h(\theta)-\frac{1}{\ell_{c}} \int_{S^{1}} h \mathrm{~d} s\right| \leqslant \frac{1}{2} \int_{S^{1}}\left|D_{s} h\right| \mathrm{d} s .
$$

Proof. Since $h(0)=h(2 \pi)$, the following equality holds:

$$
h(\theta)-h(0)=\frac{1}{2}\left(\int_{0}^{\theta} h^{\prime}(\sigma) \mathrm{d} \sigma-\int_{\theta}^{2 \pi} h^{\prime}(\sigma) \mathrm{d} \sigma\right),
$$

and hence, after integration,

$$
\frac{1}{\ell_{c}} \int_{S^{1}} h \mathrm{~d} s-h(0)=\frac{1}{2 \ell_{c}} \int_{S^{1}}\left(\int_{0}^{\theta} h^{\prime}(\sigma) \mathrm{d} \sigma-\int_{\theta}^{2 \pi} h^{\prime}(\sigma) \mathrm{d} \sigma\right) \mathrm{d} s .
$$


Next, we take the absolute value,

$$
\begin{aligned}
\left|\frac{1}{\ell_{c}} \int_{S^{1}} h \mathrm{~d} s-h(0)\right| & \leqslant \frac{1}{2 \ell_{c}} \int_{S^{1}}\left(\int_{0}^{\theta}\left|h^{\prime}(\sigma)\right| \mathrm{d} \sigma+\int_{\theta}^{2 \pi}\left|h^{\prime}(\sigma)\right| \mathrm{d} \sigma\right) \mathrm{d} s \\
& \leqslant \frac{1}{2 \ell_{c}} \int_{S^{1}}\left|h^{\prime}(\sigma)\right| \mathrm{d} \sigma \int_{S^{1}} 1 \mathrm{~d} s=\frac{1}{2} \int_{S^{1}}\left|D_{s} h\right| \mathrm{d} s .
\end{aligned}
$$

Now, we replace 0 by an arbitrary $\theta \in S^{1}$, and repeat the above steps.

This lemma permits us to prove the inequalities that we will use throughout the remainder of the paper.

LeMmA 2.14. Let $c \in \operatorname{Imm}^{2}\left(S^{1}, \mathbb{R}^{2}\right)$ and $h \in H^{2}\left(S^{1}, \mathbb{R}^{d}\right)$. Then

- $\|h\|_{L^{\infty}}^{2} \leqslant \frac{2}{\ell_{c}}\|h\|_{L^{2}(d s)}^{2}+\frac{\ell_{c}}{2}\left\|D_{s} h\right\|_{L^{2}(d s)}^{2}$,

- $\left\|D_{s} h\right\|_{L^{\infty}}^{2} \leqslant \frac{\ell_{c}}{4}\left\|D_{s}^{2} h\right\|_{L^{2}(d s)}^{2}$

- $\left\|D_{s} h\right\|_{L^{2}(d s)}^{2} \leqslant \frac{\ell_{c}^{2}}{4}\left\|D_{s}^{2} h\right\|_{L^{2}(d s)}^{2}$.

Proof. From Lemma 2.13, we obtain the inequality

$$
\|h\|_{L^{\infty}} \leqslant \frac{1}{\ell_{c}} \int_{S^{1}}|h| \mathrm{d} s+\frac{1}{2} \int_{S^{1}}\left|D_{s} h\right| \mathrm{d} s .
$$

Next, we use $(a+b)^{2} \leqslant 2 a^{2}+2 b^{2}$ and the Cauchy-Schwarz inequality

$$
\begin{aligned}
\|h\|_{L^{\infty}}^{2} & \leqslant \frac{2}{\ell_{c}^{2}}\left(\int_{S^{1}}|h| \mathrm{d} s\right)^{2}+\frac{1}{2}\left(\int_{S^{1}}\left|D_{s} h\right| \mathrm{d} s\right)^{2} \\
& \leqslant \frac{2}{\ell_{c}}\left(\int_{S^{1}}|h|^{2} \mathrm{~d} s\right)+\frac{\ell_{c}}{2}\left(\int_{S^{1}}\left|D_{s} h\right|^{2} \mathrm{~d} s\right),
\end{aligned}
$$

thus proving the first statement. To prove the second statement, we note that $\int_{S^{1}} D_{s} h \mathrm{~d} s=0$, and thus, by Lemma 2.13,

$$
\left\|D_{s} h\right\|_{L^{\infty}} \leqslant \frac{1}{2} \int_{S^{1}}\left|D_{s}^{2} h\right| \mathrm{d} s .
$$

Hence,

$$
\left\|D_{s} h\right\|_{L^{\infty}}^{2} \leqslant \frac{1}{4}\left(\int_{S^{1}}\left|D_{s}^{2} h\right| \mathrm{d} s\right)^{2} \leqslant \frac{\ell_{c}}{4}\left\|D_{s}^{2} h\right\|_{L^{2}(d s)}^{2} .
$$


To prove the third statement, we estimate

$$
\left\|D_{s} h\right\|_{L^{2}(d s)}^{2} \leqslant\left\|D_{s} h\right\|_{L^{\infty}}^{2} \int_{S^{1}} 1 \mathrm{~d} s \leqslant \frac{\ell_{c}^{2}}{4}\left\|D_{s}^{2} h\right\|_{L^{2}(d s)}^{2} .
$$

This completes the proof.

The next lemma allows us to estimate the $H^{k}(d s)$-norm using a combination of the $L^{2}(d s)$ - and the $H^{n}(d s)$-norms, without introducing constants that depend on the curve.

LEMMA 2.15. Let $n \geqslant 2, c \in \operatorname{Imm}^{n}\left(S^{1}, \mathbb{R}^{2}\right)$, and $h \in H^{n}\left(S^{1}, \mathbb{R}^{d}\right)$. Then, for $0 \leqslant k \leqslant n$,

$$
\left\|D_{s}^{k} h\right\|_{L^{2}(d s)}^{2} \leqslant\|h\|_{L^{2}(d s)}^{2}+\left\|D_{s}^{n} h\right\|_{L^{2}(d s)}^{2} .
$$

Proof. Let us write $D_{c}$ and $L^{2}(c)$ for $D_{s}$ and $L^{2}(d s)$ respectively to emphasize the dependence on the curve $c$. Since $\left\|D_{c}^{k} h\right\|_{L^{2}(c)}=\left\|D_{c \circ \varphi}^{k}(h \circ \varphi)\right\|_{L^{2}(c \circ \varphi)}$, we can assume that $c$ has a constant speed parameterization, i.e., $\left|c^{\prime}\right|=\ell_{c} / 2 \pi$. The inequality we have to show is

$$
\int_{0}^{2 \pi}\left(\frac{2 \pi}{\ell_{c}}\right)^{2 k-1}\left|h^{(k)}(\theta)\right|^{2} \mathrm{~d} \theta \leqslant \int_{0}^{2 \pi} \frac{\ell_{c}}{2 \pi}|h(\theta)|^{2}+\left(\frac{2 \pi}{\ell_{c}}\right)^{2 n-1}\left|h^{(n)}(\theta)\right|^{2} \mathrm{~d} \theta .
$$

Let $\varphi(x)=\left(2 \pi / \ell_{c}\right) x$. After a change of variables, this becomes

$$
\int_{0}^{\ell_{c}}\left|(h \circ \varphi)^{(k)}(x)\right|^{2} \mathrm{~d} x \leqslant \int_{0}^{\ell_{c}}|h \circ \varphi(x)|^{2}+\left|(h \circ \varphi)^{(n)}(x)\right|^{2} \mathrm{~d} x .
$$

Let $f=h \circ \varphi$, and assume w.l.o.g. that $f$ is $\mathbb{R}$-valued. Define $f_{k}(x)=$ $\ell_{c}^{-1 / 2} \exp \left(i\left(2 \pi k / \ell_{c}\right) x\right)$, which is an orthonormal basis of $L^{2}\left(\left[0, \ell_{c}\right], \mathbb{R}\right)$. Then $f=\sum_{k \in \mathbb{Z}} \widehat{f}(k) f_{k}$, and (3) becomes

$$
\sum_{k \in \mathbb{Z}}\left(\frac{2 \pi k}{\ell_{c}}\right)^{2 k}|\widehat{f}(k)|^{2} \leqslant \sum_{k \in \mathbb{Z}}\left[1+\left(\frac{2 \pi k}{\ell_{c}}\right)^{2 n}\right]|\widehat{f}(k)|^{2} .
$$

Since for $a \geqslant 0$ we have the inequality $a^{k} \leqslant 1+a^{n}$, the last inequality is satisfied, thus concluding the proof.

An alternative way to estimate the $H^{k}(d s)$-norm is given by the following lemma, which is the periodic version of the Gagliardo-Nirenberg inequalities (see [20]). 
LEMmA 2.16. Let $n \geqslant 2, c \in \operatorname{Imm}^{n}\left(S^{1}, \mathbb{R}^{2}\right)$, and $h \in H^{n}\left(S^{1}, \mathbb{R}^{d}\right)$. Then, for $0 \leqslant k \leqslant n$,

$$
\left\|D_{s}^{k} h\right\|_{L^{2}(d s)} \leqslant\|h\|_{L^{2}(d s)}^{1-k / n}\left\|D_{s}^{n} h\right\|_{L^{2}(d s)}^{k / n} .
$$

If $c \in \operatorname{Imm}^{2}\left(S^{1}, \mathbb{R}^{2}\right)$, the inequality also holds for $n=0,1$.

\subsection{The geodesic equation on weak Riemannian manifolds. Let $V$ be} a convenient vector space, $M \subseteq V$ an open subset, and $G$ a possibly weak Riemannian metric on $M$. Denote by $\bar{L}: T M \rightarrow(T M)^{\prime}$ the canonical map defined by

$$
G_{c}(h, k)=\left\langle\bar{L}_{c} h, k\right\rangle_{T M},
$$

with $c \in M, h, k \in T_{c} M$, and with $\langle\cdot, \cdot\rangle_{T M}$ denoting the canonical pairing between $(T M)^{\prime}$ and $T M$. We also define $H_{c}(h, h) \in\left(T_{c} M\right)^{\prime}$ via

$$
D_{c, m} G_{c}(h, h)=\left\langle H_{c}(h, h), m\right\rangle_{T M},
$$

with $D_{c, m}$ denoting the directional derivative at $c$ in direction $m$. In fact, $H$ is a smooth map,

$$
H: T M \rightarrow(T M)^{\prime}, \quad(c, h) \mapsto\left(c, H_{c}(h, h)\right) .
$$

With these definitions we can state how to calculate the geodesic equation.

LEMma 2.18. The geodesic equation-or equivalently the Levi-Civita covariant derivative - on $(M, G)$ exists if and only if $\frac{1}{2} H_{c}(h, h)-\left(D_{c, h} \bar{L}_{c}\right)(h)$ is in the image of $\bar{L}_{c}$ for all $(c, h) \in T M$, and the map

$$
T M \rightarrow T M, \quad(c, h) \mapsto \bar{L}_{c}^{-1}\left(\frac{1}{2} H_{c}(h, h)-\left(D_{c, h} \bar{L}_{c}\right)(h)\right)
$$

is smooth. In this case, the geodesic equation can be written as

$$
\begin{aligned}
c_{t} & =\bar{L}_{c}^{-1} p \\
p_{t} & =\frac{1}{2} H_{c}\left(c_{t}, c_{t}\right)
\end{aligned} \quad \text { or } \quad c_{t t}=\frac{1}{2} \bar{L}_{c}^{-1}\left(H_{c}\left(c_{t}, c_{t}\right)-\left(\partial_{t} \bar{L}_{c}\right)\left(c_{t}\right)\right) .
$$

This lemma is an adaptation of the result given in [3, 2.4.1], and the same proof can be repeated; see also [16, Section 2.4].

\section{Sobolev metrics with constant coefficients}

In this paper, we will consider Sobolev-type metrics with constant coefficients. These are metrics of the form

$$
G_{c}(h, k)=\int_{S^{1}} \sum_{j=0}^{n} a_{j}\left\langle D_{s}^{j} h, D_{s}^{j} k\right\rangle \mathrm{d} s,
$$


with $a_{j} \geqslant 0$ and $a_{0}, a_{n} \neq 0$. We call $n$ the order of the metric. The metric can be defined either on the space $\operatorname{Imm}\left(S^{1}, \mathbb{R}^{2}\right)$ of $\left(C^{\infty}\right.$-)smooth immersions or for $p \geqslant n$ on the spaces $\operatorname{Imm}^{p}\left(S^{1}, \mathbb{R}^{2}\right)$ of Sobolev $H^{p}$-immersions.

3.1. The space of smooth immersions. Let us first consider $G$ on the space of smooth immersions. The metric can be represented via the associated family of operators, $L$, which are defined by

$$
G_{c}(h, k)=\int_{S^{1}}\left\langle L_{c} h, k\right\rangle \mathrm{d} s=\int_{S^{1}}\left\langle h, L_{c} k\right\rangle \mathrm{d} s .
$$

The operator $L_{c}: T_{c} \operatorname{Imm}\left(S^{1}, \mathbb{R}^{2}\right) \rightarrow T_{c} \operatorname{Imm}\left(S^{1}, \mathbb{R}^{2}\right)$ for a Sobolev metric with constant coefficients can be calculated via integration by parts, and it is given by

$$
L_{c} h=\sum_{j=0}^{n}(-1)^{j} a_{j} D_{s}^{2 j} h .
$$

The operator $L_{c}$ is self-adjoint, positive, and hence injective. Since $L_{c}$ is elliptic, it is Fredholm $H^{k} \rightarrow H^{k-2 n}$ with vanishing index, and thus surjective. Furthermore, its inverse is smooth as well. We want to distinguish between the operator $L_{c}$ and the canonical embedding from $T_{c}$ Imm into $\left(T_{c} \mathrm{Imm}\right)^{\prime}$, which we denote by $\bar{L}_{c}$. They are related via

$$
\bar{L}_{c} h=L_{c} h \otimes \mathrm{d} s=L_{c} h \otimes\left|c^{\prime}\right| \mathrm{d} \theta .
$$

Later, we will simply write $\bar{L}_{c} h=L_{c} h\left|c^{\prime}\right|$, especially when the order of multiplication and differentiation becomes important in Sobolev spaces.

3.2. The space of Sobolev immersions. Assume that $n \geqslant 2$, and let $G$ be a Sobolev metric of order $n$. We want to extend $G$ from the space $\operatorname{Imm}\left(S^{1}, \mathbb{R}^{2}\right)$ to a smooth metric on the Sobolev completion $\operatorname{Imm}^{n}\left(S^{1}, \mathbb{R}^{2}\right)$. First, we have to look at the action of the arc length derivative and its transpose (with respect to $H^{0}(d \theta)$ ) on Sobolev spaces. Remember that we always use the $H^{n}(d \theta)$-norm on Sobolev completions. We can write $D_{s}$ as the composition $D_{s}=1 /\left|c^{\prime}\right| \circ \partial_{\theta}$, where $1 /\left|c^{\prime}\right|$ is interpreted as the multiplication operator $f \mapsto\left(1 /\left|c^{\prime}\right|\right) f$. Its transpose is $D_{s}^{*}=$ $\partial_{\theta}^{*} \circ\left(1 /\left|c^{\prime}\right|\right)^{*}=-\partial_{\theta} \circ 1 /\left|c^{\prime}\right|$. These operators are smooth in the following sense.

LemmA 3.3. Let $n \geqslant 2$ and $k \in \mathbb{Z}$ with $|k| \leqslant n-1$. Then the maps

$$
\begin{aligned}
& D_{s}: \operatorname{Imm}^{n}\left(S^{1}, \mathbb{R}^{2}\right) \times H^{k+1}\left(S^{1}, \mathbb{R}^{d}\right) \rightarrow H^{k}\left(S^{1}, \mathbb{R}^{d}\right), \quad(c, h) \mapsto D_{s} h=\frac{1}{\left|c^{\prime}\right|} h^{\prime} \\
& D_{s}^{*}: \operatorname{Imm}^{n}\left(S^{1}, \mathbb{R}^{2}\right) \times H^{k}\left(S^{1}, \mathbb{R}^{d}\right) \rightarrow H^{k-1}\left(S^{1}, \mathbb{R}^{d}\right), \quad(c, h) \mapsto D_{s}^{*} h=-\left(\frac{1}{\left|c^{\prime}\right|} h\right)^{\prime}
\end{aligned}
$$

are smooth. 
Proof. For $n \geqslant 2$, the map $c \mapsto 1 /\left|c^{\prime}\right|$ is the composition of the following smooth maps:

$$
\begin{aligned}
& \operatorname{Imm}^{n}\left(S^{1}, \mathbb{R}^{2}\right) \rightarrow\{f: f>0\} \subset H^{n-1}\left(S^{1}, \mathbb{R}\right) \rightarrow H^{n-1}\left(S^{1}, \mathbb{R}\right) \\
& c \quad \mapsto \quad\left|c^{\prime}\right| \quad \mapsto \quad \frac{1}{\left|c^{\prime}\right|} .
\end{aligned}
$$

Since $1 /\left|c^{\prime}\right| \in H^{n-1}\left(S^{1}, \mathbb{R}^{2}\right)$, Lemma 2.5 concludes the proof.

Using Lemma 3.3, we see that

$$
G_{c}(h, h)=\int_{S^{1}} \sum_{k=0}^{n} a_{k}\left\langle D_{s}^{k} h, D_{s}^{k} h\right\rangle \mathrm{d} s
$$

is well defined for $(c, h) \in T \operatorname{Imm}^{n}\left(S^{1}, \mathbb{R}^{2}\right)$. As the tangent bundle is isomorphic to $T \operatorname{Imm}^{n}\left(S^{1}, \mathbb{R}^{2}\right) \cong \operatorname{Imm}^{n}\left(S^{1}, \mathbb{R}^{2}\right) \times H^{n}\left(S^{1}, \mathbb{R}^{2}\right)$, we can also write the metric as

$$
G_{c}(h, h)=\left\langle\sum_{k=0}^{n} a_{k}\left(D_{s}^{k}\right)^{*}\left|c^{\prime}\right| D_{s}^{k} h, h\right\rangle_{H^{-n} \times H^{n}} .
$$

Again we note that $\left|c^{\prime}\right|$ has to be interpreted as the multiplication operator $f \mapsto$ $\left|c^{\prime}\right| f$ on the spaces $H^{k}$ with $|k| \leqslant n-1$. Thus the operator $\bar{L}_{c}: H^{n} \rightarrow H^{-n}$ is given by

$$
\bar{L}_{c}=\sum_{k=0}^{n} a_{k}\left(D_{s}^{k}\right)^{*} \circ\left|c^{\prime}\right| \circ D_{s}^{k} .
$$

While it is tempting to 'simplify' the expression for $\bar{L}_{c}$ using the identity

$$
D_{s}^{*} \circ\left|c^{\prime}\right|=-\left|c^{\prime}\right| \circ D_{s},
$$

one has to be careful, since the identity is only valid when interpreted as an operator $H^{k} \rightarrow H^{k-1}$ with $-n+2 \leqslant k \leqslant n-1$. The left-hand side, however, makes sense also for $k=-n+1$. Thus we have the operator

$$
\left(D_{s}^{n}\right)^{*} \circ\left|c^{\prime}\right|: L^{2} \rightarrow H^{-n},
$$

but the domain has to be at least $H^{1}$ for the operator

$$
(-1)^{n}\left|c^{\prime}\right| \circ D_{s}^{n}: H^{1} \rightarrow H^{-n+1} .
$$

So the expression

$$
\bar{L}_{c} h=\sum_{k=0}^{n}(-1)^{k} a_{k}\left|c^{\prime}\right| D_{s}^{2 k} h
$$

is only valid when we restrict $\bar{L}_{c}$ to $H^{n+1}$, i.e., $\bar{L}_{c}: H^{n+1} \rightarrow H^{-n+1}$. 
3.4. The geodesic equation. By Lemma 2.18, we need to calculate $H_{c}(h, h)$. This is achieved in the following lemma.

LEMmA 3.5. Let $n \geqslant 2$, and let $G$ be a Sobolev metric of order $n$. On $\operatorname{Imm}^{n}\left(S^{1}\right.$, $\mathbb{R}^{2}$ ), we have $H_{c}(h, h)=-a_{0}\left|c^{\prime}\right| D_{s}(\langle h, h\rangle v)-\sum_{k=1}^{n} \sum_{j=1}^{2 k-1}(-1)^{k+j} a_{k} D_{s}^{*} \circ\left(\left|c^{\prime}\right|\left\langle D_{s}^{2 k-j} h, D_{s}^{j} h\right\rangle v\right)$.

On $\operatorname{Imm}^{p}\left(S^{1}, \mathbb{R}^{2}\right)$ with $p \geqslant n+1$ as well as $\operatorname{Imm}\left(S^{1}, \mathbb{R}^{2}\right)$, we have the equivalent expression,

$$
\begin{aligned}
H_{c}(h, h)= & \left(-2\left\langle L_{c} h, D_{s} h\right\rangle v-a_{0}\langle h, h\rangle \kappa n\right. \\
& \left.+\sum_{k=1}^{n} \sum_{j=1}^{2 k-1}(-1)^{k+j} a_{k}\left\langle D_{s}^{2 k-j} h, D_{s}^{j} h\right\rangle \kappa n\right) \otimes \mathrm{d} s .
\end{aligned}
$$

Proof. For $k \geqslant 1$, the variation of the $k$ th arc length derivative is

$$
D_{c, m} D_{s}^{k} h=-\sum_{j=1}^{k} D_{s}^{k-j}\left(\left\langle D_{s} m, v\right\rangle D_{s}^{j} h\right)
$$

and the formula is valid for $(c, m) \in T \operatorname{Imm}^{n}\left(S^{1}, \mathbb{R}^{2}\right)$ and $h \in H^{-n+k}\left(S^{1}, \mathbb{R}^{d}\right)$. So

$$
\begin{aligned}
D_{c, m} G_{c}(h, h)= & \int_{S^{1}} \sum_{k=0}^{n} a_{k}\left\langle D_{s}^{k} h, D_{s}^{k} h\right\rangle\left\langle D_{s} m, v\right\rangle\left|c^{\prime}\right| \\
& +2 \sum_{k=1}^{n} a_{k}\left\langle D_{s}^{k} h, D_{c, m} D_{s}^{k} h\right\rangle\left|c^{\prime}\right| \mathrm{d} \theta \\
= & \left\langle\sum_{k=0}^{n} a_{k}\left|c^{\prime}\right|\left\langle D_{s}^{k} h, D_{s}^{k}\right\rangle v, D_{s} m\right\rangle_{H^{-n+1} \times H^{n-1}} \\
& \quad-2 \sum_{k=1}^{n} \sum_{j=1}^{k} a_{k}\left\langle\left|c^{\prime}\right| D_{s}^{k} h, D_{s}^{k-j}\left\langle D_{s} m, v\right\rangle D_{s}^{j} h\right\rangle_{H^{-n+k} \times H^{n-k}} .
\end{aligned}
$$

Each term in the second sum is equal to

$$
\begin{aligned}
\left\langle\left|c^{\prime}\right| D_{s}^{k} h, D_{s}^{k-j}\left\langle D_{s} m, v\right\rangle\right. & \left.D_{s}^{j} h\right\rangle_{H^{-n+k} \times H^{n-k}} \\
& =\left\langle\left(D_{s}^{k-j}\right)^{*}\left|c^{\prime}\right| D_{s}^{k} h,\left\langle D_{s} m, v\right\rangle D_{s}^{j} h\right\rangle_{H^{-n+j} \times H^{n-j}}
\end{aligned}
$$




$$
\begin{aligned}
& =(-1)^{k-j}\left\langle\left|c^{\prime}\right| D_{s}^{2 k-j} h,\left\langle D_{s} m, v\right\rangle D_{s}^{j} h\right\rangle_{H^{-n+j} \times H^{n-j}} \\
& =(-1)^{k-j}\left\langle\left|c^{\prime}\right|\left\langle D_{s}^{2 k-j} h, D_{s}^{j} h\right\rangle v, D_{s} m\right\rangle_{H^{-n+1} \times H^{n-1}}
\end{aligned}
$$

So

$$
\begin{aligned}
H_{c}(h, h) & \\
= & \sum_{k=0}^{n} a_{k} D_{s}^{*} \circ\left(\left|c^{\prime}\right|\left\langle D_{s}^{k} h, D_{s}^{k} h\right\rangle v\right) \\
& \quad-2 \sum_{k=1}^{n} \sum_{j=1}^{k}(-1)^{k-j} a_{k} D_{s}^{*} \circ\left(\left|c^{\prime}\right|\left\langle D_{s}^{2 k-j} h, D_{s}^{j} h\right\rangle v\right) \\
= & -a_{0}\left|c^{\prime}\right| D_{s}(\langle h, h\rangle v)-\sum_{k=1}^{n} \sum_{j=1}^{2 k-1}(-1)^{k+j} a_{k} D_{s}^{*} \circ\left(\left|c^{\prime}\right|\left\langle D_{s}^{2 k-j} h, D_{s}^{j} h\right\rangle v\right) .
\end{aligned}
$$

This proves the first formula.

If $(c, h) \in T \operatorname{Imm}^{p}\left(S^{1}, \mathbb{R}^{2}\right)$ with $p \geqslant 1$, we can commute $D_{s}^{*} \circ\left|c^{\prime}\right|=-\left|c^{\prime}\right| \circ D_{s}$ to obtain

$$
H_{c}(h, h)=-a_{0}\left|c^{\prime}\right| D_{s}(\langle h, h\rangle v)+\sum_{k=1}^{n} \sum_{j=1}^{2 k-1}(-1)^{k+j} a_{k}\left|c^{\prime}\right| D_{s}\left(\left\langle D_{s}^{2 k-j} h, D_{s}^{j} h\right\rangle v\right) .
$$

Parts of the expression simplify as follows:

$$
\begin{aligned}
\sum_{k=1}^{n} & \sum_{j=1}^{2 k-1}(-1)^{k+j} a_{k} D_{s}\left(\left\langle D_{s}^{2 k-j} h, D_{s}^{j} h\right\rangle\right)-a_{0} D_{s}(\langle h, h\rangle) \\
= & \sum_{k=1}^{n} \sum_{j=1}^{2 k-1}(-1)^{k+j} a_{k}\left(\left\langle D_{s}^{2 k-j+1} h, D_{s}^{j} h\right\rangle+\left\langle D_{s}^{2 k-j} h, D_{s}^{j+1} h\right\rangle\right)-2 a_{0}\left\langle h, D_{s} h\right\rangle \\
= & \sum_{k=1}^{n} a_{k}\left(\sum_{j=0}^{2 k-2}(-1)^{k+j+1}\left\langle D_{s}^{2 k-j} h, D_{s}^{j} h\right\rangle+\sum_{j=1}^{2 k-1}(-1)^{k+j}\left\langle D_{s}^{2 k-j} h, D_{s}^{j+1} h\right\rangle\right) \\
& -2 a_{0}\left\langle h, D_{s} h\right\rangle \\
= & \sum_{k=1}^{n}(-1)^{k+1} 2 a_{k}\left\langle D_{s}^{2 k} h, D_{s} h\right\rangle-2 a_{0}\left\langle h, D_{s} h\right\rangle \\
= & -2\left\langle L_{c} h, D_{s} h\right\rangle,
\end{aligned}
$$

and by collecting the remaining terms we arrive at the desired result. 
Now that we have computed $H_{c}(h, h)$, we can write the geodesic equation of the metric $G$. It is

$$
\begin{aligned}
\partial_{t}\left(\bar{L}_{c} c_{t}\right)= & -\frac{a_{0}}{2}\left|c^{\prime}\right| D_{s}\left(\left\langle c_{t}, c_{t}\right\rangle v\right) \\
& -\sum_{k=1}^{n} \sum_{j=1}^{2 k-1}(-1)^{k+j} \frac{a_{k}}{2} D_{s}^{*} \circ\left(\left|c^{\prime}\right|\left\langle D_{s}^{2 k-j} c_{t}, D_{s}^{j} c_{t}\right\rangle v\right) .
\end{aligned}
$$

3.6. Local well-posedness. It has been shown in [18, Theorem 4.3] that the geodesic equation of a Sobolev metric is well-posed on $\operatorname{Imm}^{p}\left(S^{1}, \mathbb{R}^{2}\right)$ for $p \geqslant$ $2 n+1$. For a metric of order $n \geqslant 2$, we extend the result to $p \geqslant n$. This will later simplify the proof of geodesic completeness.

THEOREM 3.7. Let $n \geqslant 2$ and $p \geqslant n$, and let $G$ be a Sobolev metric of order $n$ with constant coefficients. Then the geodesic equation (5) has unique local solutions in the space $\operatorname{Imm}^{p}\left(S^{1}, \mathbb{R}^{2}\right)$ of Sobolev $H^{p}$-immersions. The solutions depend $C^{\infty}$-smoothly on $t$ and the initial conditions. The domain of existence (in t) is uniform in $p$, and thus the geodesic equation also has local solutions in $\operatorname{Imm}\left(S^{1}, \mathbb{R}^{2}\right)$, the space of smooth immersions.

Proof. Fix $p \geqslant n$. For the geodesic equation to exist, we need to verify the assumptions in Lemma 2.18. We first note that $\bar{L}_{c}$ is a map $\bar{L}_{c}: H^{p} \rightarrow H^{p-2 n}$. By inspecting (4), we see that $H_{c}(h, h) \in H^{p-2 n}$ as well. Thus it remains to show that $\bar{L}_{c}$ maps $H^{p}$ onto $H^{p-2 n}$, and that the inverse is smooth. This is shown in Lemma 3.8.

Regarding local existence, we rewrite the geodesic equation as a differential equation on $T \operatorname{Imm}^{n}\left(S^{1}, \mathbb{R}^{2}\right)$,

$$
\begin{aligned}
c_{t} & =u \\
u_{t} & =\frac{1}{2} \bar{L}_{c}^{-1}\left(H_{c}(u, u)-\left(D_{c, u} \bar{L}_{c}\right)(u)\right) .
\end{aligned}
$$

This is a smooth ODE on a Hilbert space, and therefore by the theorem of PicardLindelöf it has local solutions that depend smoothly on $t$ and the initial conditions. That the intervals of existence are uniform in the Sobolev order $p$ can be found in [3, Appendix A]. The result goes back to [9, Theorem 12.1], and a different proof can be found in [18].

The following lemma shows that the operator $\bar{L}_{c}$ has a smooth inverse on appropriate Sobolev spaces. For $p=n$, we can use Lemma 5.1 and the LaxMilgram lemma to show that $\bar{L}_{c}: H^{n} \rightarrow H^{-n}$ is invertible. For $p>n$, more 
work is necessary. Although $\bar{L}_{c}$ is an elliptic positive differential operator, it has nonsmooth coefficients. In fact, since $\left|c^{\prime}\right| \in H^{n-1}$, some of the coefficients are only distributions. To overcome this, we will exploit the reparameterization invariance of the metric to transform $\bar{L}_{c}$ into a differential operator with constant coefficients.

LEMmA 3.8. Let $n \geqslant 2$, and let $G$ be a Sobolev metric of order $n$. For $p \geqslant n$ and $c \in \operatorname{Imm}^{p}\left(S^{1}, \mathbb{R}^{2}\right)$, the associated operators

$$
\bar{L}_{c}: H^{p}\left(S^{1}, \mathbb{R}^{d}\right) \rightarrow H^{p-2 n}\left(S^{1}, \mathbb{R}^{d}\right),
$$

are isomorphisms, and the map

$$
\bar{L}^{-1}: \operatorname{Imm}^{p}\left(S^{1}, \mathbb{R}^{2}\right) \times H^{p-2 n}\left(S^{1}, \mathbb{R}^{d}\right) \rightarrow H^{p}\left(S^{1}, \mathbb{R}^{d}\right), \quad(c, h) \mapsto \bar{L}_{c}^{-1} h
$$

is smooth.

Proof. Given a curve $c \in \operatorname{Imm}^{p}\left(S^{1}, \mathbb{R}^{2}\right)$, we can write it as $c=d \circ \psi$, where $d$ has constant speed, $\left|d^{\prime}\right|=\ell_{c} / 2 \pi$, and $\psi$ is a diffeomorphism of $S^{1}$. The pair $(d, \psi)$ is determined only up to rotations; we can remove the ambiguity by requiring that $c(0)=d(0)$. Then $\psi$ is given by

$$
\psi(\theta)=\frac{2 \pi}{\ell_{c}} \int_{0}^{\theta}\left|c^{\prime}(\sigma)\right| \mathrm{d} \sigma .
$$

Concerning regularity, we have $\psi$ and $\psi^{-1} \in H^{p}\left(S^{1}, S^{1}\right)$; thus $\psi \in \mathcal{D}^{p}\left(S^{1}\right)$, and $d \in \operatorname{Imm}^{p}\left(S^{1}, \mathbb{R}^{2}\right)$.

The reparameterization invariance of the metric $G$ implies that

$$
\left\langle\bar{L}_{c} h, m\right\rangle_{H^{-p} \times H^{p}}=\left\langle\bar{L}_{c \circ \psi^{-1}}\left(h \circ \psi^{-1}\right), m \circ \psi^{-1}\right\rangle_{H^{-p} \times H^{p}} .
$$

Introduce the notation $R_{\varphi}(h)=h \circ \varphi$. If $\varphi \in \mathcal{D}^{p}\left(S^{1}\right)$ is a diffeomorphism, the map $R_{\varphi}$ is an invertible linear map $R_{\varphi}: H^{p} \rightarrow H^{p}$, by Lemma 2.6. Furthermore, by Lemma 2.7, the transpose $R_{\varphi}^{*}$ is an invertible map $R_{\varphi}^{*}: H^{p-2 n} \rightarrow H^{p-2 n}$. Thus we get

$$
\bar{L}_{c} h=R_{\psi^{-1}}^{*} \circ \bar{L}_{d} \circ R_{\psi^{-1}}(h) .
$$

Because $\left|d^{\prime}\right|=\ell_{c} / 2 \pi$, the operator $\bar{L}_{d}$ is equal to

$$
\bar{L}_{d}=\sum_{k=0}^{n}(-1)^{k} a_{k}\left(\frac{2 \pi}{\ell_{c}}\right)^{2 k-1} \partial_{\theta}^{2 k} .
$$

This is a positive elliptic differential operator with constant coefficients, and thus $\bar{L}_{d}: H^{p} \rightarrow H^{p-2 n}$ is invertible. Thus the composition $\bar{L}_{c}: H^{p} \rightarrow H^{p-2 n}$ 
is invertible. Smoothness of $(c, h) \mapsto \bar{L}_{c}^{-1} h$ follows from the smoothness of $(c, h) \mapsto \bar{L}_{c} h$ and the implicit function theorem on Banach spaces.

The remainder of the paper will be concerned with the analysis of the geodesic distance function induced by Sobolev metrics. These results will be used to show that geodesics for metrics of order 2 and higher exist for all times.

\section{Lower bounds on the geodesic distance}

To prepare the proof of geodesic completeness, we first need to use the geodesic distance to estimate quantities that are derived from the curve and that appear in the geodesic equation. These include the length $\ell_{c}$, curvature $\kappa$, and its derivatives $D_{s}^{k} \kappa$, as well as the length element $\left|c^{\prime}\right|$ and its derivatives $D_{s}^{k} \log \left|c^{\prime}\right|$. We want to show that they are bounded on metric balls of a Sobolev metric of sufficiently high order.

We start with the length $\ell_{c}$. The argument given in [18, Section 4.7] can be used to show the following slightly stronger statement.

LEMMA 4.1. Let the metric $G$ on $\operatorname{Imm}\left(S^{1}, \mathbb{R}^{2}\right)$ satisfy

$$
\int_{S^{1}}\left\langle D_{s} h, v\right\rangle^{2} \mathrm{~d} s \leqslant A G_{c}(h, h)
$$

for some $A>0$. Then we have the estimate

$$
\left\|\sqrt{\left|c_{1}^{\prime}\right|}-\sqrt{\left|c_{2}^{\prime}\right|}\right\|_{L^{2}(d \theta)} \leqslant \frac{\sqrt{A}}{2} \operatorname{dist}^{G}\left(c_{1}, c_{2}\right),
$$

and in particular the function $\sqrt{\mid \overline{c^{\prime} \mid}}:\left(\operatorname{Imm}\left(S^{1}, \mathbb{R}^{2}\right)\right.$, $\left.\operatorname{dist}^{G}\right) \rightarrow L^{2}\left(S^{1}, \mathbb{R}\right)$ is Lipschitz.

Proof. Take two curves $c_{1}, c_{2} \in \operatorname{Imm}\left(S^{1}, \mathbb{R}^{2}\right)$, and let $c(t, \theta)$ be a smooth path between them. Then the following relation holds pointwise for each $\theta \in S^{1}$ :

$$
\sqrt{\left|c_{2}^{\prime}\right|}(\theta)-\sqrt{\left|c_{1}^{\prime}\right|}(\theta)=\int_{0}^{1} \partial_{t}\left(\sqrt{\left|c^{\prime}\right|}\right)(t, \theta) \mathrm{d} t .
$$

The derivative $\partial_{t} \sqrt{\left|c^{\prime}\right|}$ is given by

$$
\partial_{t} \sqrt{\left|c^{\prime}\right|}=\frac{1}{2}\left\langle D_{s} c_{t}, v\right\rangle \sqrt{\left|c^{\prime}\right|},
$$

and so

$$
\begin{aligned}
\left\|\sqrt{\left|c_{1}^{\prime}\right|}-\sqrt{\left|c_{2}^{\prime}\right|}\right\|_{L^{2}(d \theta)} & \leqslant \frac{1}{2} \int_{0}^{1}\left\|\left\langle D_{s} c_{t}, v\right\rangle \sqrt{\left|c^{\prime}\right|}\right\|_{L^{2}(d \theta)} \mathrm{d} t \\
& \leqslant \frac{1}{2} \int_{0}^{1}\left\|\left\langle D_{s} c_{t}, v\right\rangle\right\|_{L^{2}(d s)} \mathrm{d} t
\end{aligned}
$$




$$
\begin{aligned}
& \leqslant \frac{\sqrt{A}}{2} \int_{0}^{1} \sqrt{G_{c}\left(c_{t}, c_{t}\right)} \mathrm{d} t \\
& \leqslant \frac{\sqrt{A}}{2} \operatorname{Len}^{G}(c) .
\end{aligned}
$$

Since this estimate holds for every smooth path $c$, by taking the infimum we obtain

$$
\left\|\sqrt{\left|c_{1}^{\prime}\right|}-\sqrt{\left|c_{2}^{\prime}\right|}\right\|_{L^{2}} \leqslant \frac{\sqrt{A}}{2} \inf _{c} \operatorname{Len}^{G}(c)=\frac{\sqrt{A}}{2} \operatorname{dist}^{G}\left(c_{1}, c_{2}\right) .
$$

We recover the statement of [18, Section 4.7] by applying the reverse triangle inequality. The following corollary is a disguised version of the fact that, on a normed space, the norm function is Lipschitz.

COROLlary 4.2. If the metric $G$ on $\operatorname{Imm}\left(S^{1}, \mathbb{R}^{2}\right)$ satisfies

$$
\int_{S^{1}}\left\langle D_{s} h, v\right\rangle^{2} \mathrm{~d} s \leqslant A G_{c}(h, h)
$$

for some $A>0$, then the function $\sqrt{\ell_{c}}:\left(\operatorname{Imm}\left(S^{1}, \mathbb{R}^{2}\right)\right.$, dist $\left.{ }^{G}\right) \rightarrow \mathbb{R}_{>0}$ is Lipschitz.

Proof. The statement follows from

$$
\ell_{c}=\int_{S^{1}}\left|c^{\prime}(\theta)\right| \mathrm{d} \theta=\left\|\sqrt{\left|c^{\prime}\right|}\right\|_{L^{2}(d \theta)}^{2},
$$

and the inequality

$$
\begin{aligned}
\left|\sqrt{\ell_{c_{1}}}-\sqrt{\ell_{c_{2}}}\right| & =\left|\left\|\sqrt{\left|c_{1}^{\prime}\right|}\right\|_{L^{2}(d \theta)}-\| \sqrt{\left|c_{2}^{\prime}\right|}\right|_{L^{2}(d \theta)} \mid \\
& \leqslant\left\|\sqrt{\left|c_{1}^{\prime}\right|}-\sqrt{\left|c_{2}^{\prime}\right|}\right\|_{L^{2}(d \theta)} \leqslant \frac{\sqrt{A}}{2} \operatorname{dist}^{G}\left(c_{1}, c_{2}\right) .
\end{aligned}
$$

REMARK 4.3. Lemma 4.1 and Corollary 4.2 apply in particular to Sobolev metrics of order $n \geqslant 1$. For $n=1$, this is clear from $\left\langle D_{s} h, v\right\rangle^{2} \leqslant\left|D_{s} h\right|^{2}$. For $n \geqslant 2$, we use Lemma 2.15 to estimate

$$
\begin{aligned}
\int_{S^{1}}\left\langle D_{s} h, v\right\rangle^{2} \mathrm{~d} s & \leqslant\left\|D_{s} h\right\|_{L^{2}(d s)}^{2} \leqslant\|h\|_{L^{2}(d s)}^{2}+\left\|D_{s}^{n} h\right\|_{L^{2}(d s)}^{2} \\
& \leqslant \max \left(a_{0}^{-1}, a_{n}^{-1}\right) G_{c}(h, h) .
\end{aligned}
$$

We could also have used Lemma 2.16, 


$$
\begin{aligned}
\int_{S^{1}}\left\langle D_{s} h, v\right\rangle^{2} \mathrm{~d} s & \leqslant\left\|D_{s} h\right\|_{L^{2}(d s)}^{2} \leqslant\|h\|_{L^{2}(d s)}^{2-2 / n}\left\|D_{s}^{n} h\right\|_{L^{2}(d s)}^{2 / n} \\
& \leqslant a_{0}^{(1-n) / n} a_{n}^{-1 / n} G_{c}(h, h),
\end{aligned}
$$

to reach the same conclusion.

The following lemma shows a similar statement for $\ell_{c}^{-1 / 2}$. We do not get global Lipschitz continuity; instead, the function $\ell_{c}^{-1 / 2}$ is Lipschitz on every metric ball. This implies that $\ell_{c}^{-1}$ is bounded on every metric ball. We will show later in Corollary 4.11 that the pointwise quantities $\left|c^{\prime}(\theta)\right|$ and $\left|c^{\prime}(\theta)\right|^{-1}$ are also bounded on metric balls.

LEMMA 4.4. Let the metric $G$ on $\operatorname{Imm}\left(S^{1}, \mathbb{R}^{2}\right)$ satisfy

$$
\int_{S^{1}}|h|^{2}+\left|D_{s}^{n} h\right|^{2} \mathrm{~d} s \leqslant A G_{c}(h, h)
$$

for some $n \geqslant 2$ and some $A>0$. Given $c_{0} \in \operatorname{Imm}\left(S^{1}, \mathbb{R}^{2}\right)$ and $N>0$, there exists a constant $C=C\left(c_{0}, N\right)$ such that, for all $c_{1}, c_{2} \in \operatorname{Imm}\left(S^{1}, \mathbb{R}^{2}\right)$ with $\operatorname{dist}^{G}\left(c_{0}\right.$, $\left.c_{i}\right)<N, i=1,2$, we have

$$
\left|\ell_{c_{1}}^{-1 / 2}-\ell_{c_{2}}^{-1 / 2}\right| \leqslant C\left(c_{0}, N\right) \operatorname{dist}^{G}\left(c_{1}, c_{2}\right) .
$$

In particular, the function $\ell_{c}^{-1 / 2}:\left(\operatorname{Imm}\left(S^{1}, \mathbb{R}^{2}\right)\right.$, dist $\left.{ }^{G}\right) \rightarrow \mathbb{R}_{>0}$ is Lipschitz on every metric ball.

Proof. Fix $c_{1}, c_{2}$ with $\operatorname{dist}^{G}\left(c_{0}, c_{i}\right)<N$, and let $c(t, \theta)$ be a path between them, such that $\operatorname{dist}^{G}\left(c_{0}, c(t)\right)<2 N$. Then

$$
\partial_{t}\left(\ell_{c}^{-1 / 2}\right)=-\frac{1}{2} \ell_{c}^{-3 / 2} \int_{S^{1}}\left\langle D_{s} c_{t}, v\right\rangle\left|c^{\prime}\right| \mathrm{d} \theta,
$$

and, by taking absolute values,

$$
\begin{aligned}
\left|\partial_{t}\left(\ell_{c}^{-1 / 2}\right)\right| & \leqslant \frac{1}{2} \ell_{c}^{-3 / 2} \int_{S^{1}}\left|\left\langle D_{s} c_{t}, v\right\rangle\right|\left|c^{\prime}\right| \mathrm{d} \theta \\
& \leqslant \frac{1}{2} \ell_{c}^{-3 / 2} \sqrt{\int_{S^{1}}\left|c^{\prime}\right| \mathrm{d} \theta} \sqrt{\int_{S^{1}}\left\langle D_{s} c_{t}, v\right\rangle^{2}\left|c^{\prime}\right| \mathrm{d} \theta} \\
& \leqslant \frac{1}{2} \ell_{c}^{-1}\left\|D_{s} c_{t}\right\|_{L^{2}(d s)} \leqslant \frac{1}{2} \ell_{c}^{-1}\left(\frac{\ell_{c}}{2}\right)^{n-1}\left\|D_{s}^{n} c_{t}\right\|_{L^{2}(d s)} \quad \text { by } 2.14 \\
& \leqslant 2^{-n} \ell_{c}^{n-2} \sqrt{A} \sqrt{G_{c}\left(c_{t}, c_{t}\right)} .
\end{aligned}
$$


By Corollary 4.2, the length $\ell_{c}$ is bounded along the path $c(t, \theta)$, and since $n \geqslant 2$ so is $\ell_{c}^{n-2}$. Thus

$$
\begin{aligned}
&\left|\ell_{c_{1}}^{-1 / 2}-\ell_{c_{2}}^{-1 / 2}\right| \leqslant \int_{0}^{1}\left|\partial_{t}\left(\ell_{c}^{-1 / 2}\right)\right| \mathrm{d} t \\
& \leqslant 2^{-n} \sqrt{A} \int_{0}^{1} \ell_{c}^{n-2} \sqrt{G_{c}\left(c_{t}, c_{t}\right)} \mathrm{d} t \\
& \lesssim c_{0}, N \\
& \operatorname{Len}^{G}(c) ; \quad \text { see } 2.8 \text { for notation. }
\end{aligned}
$$

After taking the infimum over all paths connecting $c_{1}$ and $c_{2}$, we obtain

$$
\left|\ell_{c_{1}}^{-1 / 2}-\ell_{c_{2}}^{-1 / 2}\right| \lesssim c_{0}, N \operatorname{dist}^{G}\left(c_{1}, c_{2}\right)
$$

REMARK. We can compute the constant $C=C\left(c_{0}, N\right)$ in Lemma 4.4 explicitly. Indeed, from

$$
\left|\ell_{c_{1}}^{-1 / 2}-\ell_{c_{2}}^{-1 / 2}\right| \leqslant 2^{-n} \sqrt{A} \int_{0}^{1} \ell_{c}^{n-2} \sqrt{G_{c}\left(c_{t}, c_{t}\right)} \mathrm{d} t,
$$

we obtain, following the proof,

$$
\left|\ell_{c_{1}}^{-1 / 2}-\ell_{c_{2}}^{-1 / 2}\right| \leqslant 2^{-n} \sqrt{A}\left(\sup _{\operatorname{dist}^{G}\left(c, c_{0}\right)<N} \ell_{c}^{n-2}\right) \operatorname{dist}^{G}\left(c_{1}, c_{2}\right) .
$$

Now, using (6), we can estimate $\ell_{c}$ via

$$
\sqrt{\ell_{c}} \leqslant \sqrt{\ell_{c_{0}}}+\left|\sqrt{\ell_{c}}-\sqrt{\ell_{c_{0}}}\right| \leqslant \sqrt{\ell_{c_{0}}}+\frac{1}{2} \sqrt{A} \operatorname{dist}^{G}\left(c, c_{0}\right) \leqslant \sqrt{\ell_{c_{0}}}+\frac{1}{2} \sqrt{A} N .
$$

Thus we can use

$$
C\left(c_{0}, N\right)=2^{-n} \sqrt{A}\left(\sqrt{\ell_{c_{0}}}+\frac{1}{2} \sqrt{A} N\right)^{2 n-4}
$$

for the constant.

COROLlary 4.5. Let $G$ satisfy the assumptions of Lemma 4.4. Then $\ell_{c}^{-1}$ is bounded on every metric ball of $\left(\operatorname{Imm}\left(S^{1}, \mathbb{R}^{2}\right)\right.$, dist $\left.{ }^{G}\right)$.

Proof. Fix $c_{0} \in \operatorname{Imm}\left(S^{1}, \mathbb{R}^{2}\right)$ and $N>0$, and let $c \in \operatorname{Imm}\left(S^{1}, \mathbb{R}^{2}\right)$ with $\operatorname{dist}^{G}\left(c_{0}\right.$, $c)<N$. Then

$$
\ell_{c}^{-1 / 2} \leqslant \ell_{c_{0}}^{-1 / 2}+\left|\ell_{c_{0}}^{-1 / 2}-\ell_{c}^{-1 / 2}\right| \lesssim_{c_{0}, N} \ell_{c_{0}}+\operatorname{dist}^{G}\left(c_{0}, c\right) \lesssim_{c_{0}, N} 1,
$$

and thus $\ell_{c}^{-1 / 2}$ is bounded on metric balls, which implies that $\ell_{c}^{-1}$ is bounded as well. 
The variations of the turning angle $\alpha$ and of $\log \left|c^{\prime}\right|$ are given by

$$
\begin{aligned}
D_{c, h}\left(\log \left|c^{\prime}\right|\right) & =\left\langle D_{s} h, v\right\rangle \\
D_{c, h} \alpha & =\left\langle D_{s} h, n\right\rangle .
\end{aligned}
$$

As a preparation for the proof of Theorem 4.7, we compute explicit expressions for the variations of their derivatives.

LEMMA 4.6. Let $c \in \operatorname{Imm}\left(S^{1}, \mathbb{R}^{2}\right), h \in T_{c} \operatorname{Imm}\left(S^{1}, \mathbb{R}^{2}\right)$, and $k \geqslant 0$. Then

$$
\begin{gathered}
D_{c, h}\left(D_{s}^{k} \log \left|c^{\prime}\right|\right)=D_{s}^{k}\left\langle D_{s} h, v\right\rangle-\sum_{j=0}^{k-1}\left(\begin{array}{c}
k \\
j+1
\end{array}\right)\left(D_{s}^{k-j} \log \left|c^{\prime}\right|\right) D_{s}^{j}\left\langle D_{s} h, v\right\rangle \\
D_{c, h}\left(D_{s}^{k} \alpha\right)=D_{s}^{k}\left\langle D_{s} h, n\right\rangle-\sum_{j=0}^{k-1}\left(\begin{array}{c}
k \\
j+1
\end{array}\right)\left(D_{s}^{k-j} \alpha\right) D_{s}^{j}\left\langle D_{s} h, v\right\rangle .
\end{gathered}
$$

Proof. Recall Lemma 2.3: if $F: \operatorname{Imm}\left(S^{1}, \mathbb{R}^{2}\right) \rightarrow C^{\infty}\left(S^{1}, \mathbb{R}^{d}\right)$ is smooth, then

$$
D_{c, h}\left(D_{s} \circ F\right)=D_{s}\left(D_{c, h} F\right)-\left\langle D_{s} h, v\right\rangle D_{s} F(c) .
$$

For $k=0$, by Section 2.2, we have

$$
\begin{aligned}
D_{c, h}\left(\log \left|c^{\prime}\right|\right) & =\left\langle D_{s} h, v\right\rangle, \quad D_{c, h} \alpha=\left\langle D_{s} h, n\right\rangle, \quad D_{c, h} D_{s}=-\left\langle D_{s} h, v\right\rangle D_{s}, \\
D_{c, h}\left(D_{s}^{k}\right) & =-\sum_{j=0}^{k-1} D_{s}^{j} \circ\left\langle D_{s} h, v\right\rangle \circ D_{s}^{k-j} .
\end{aligned}
$$

Thus we get

$$
D_{c, h}\left(D_{s}^{k} \log \left|c^{\prime}\right|\right)=D_{s}^{k}\left\langle D_{s} h, v\right\rangle-\sum_{j=0}^{k-1} D_{s}^{j}\left(\left\langle D_{s} h, v\right\rangle\left(D_{s}^{k-j} \log \left|c^{\prime}\right|\right)\right) .
$$

Next, we use the identity [21, (26.3.7)]

$$
\sum_{j=i}^{k-1}\left(\begin{array}{l}
j \\
i
\end{array}\right)=\left(\begin{array}{c}
k \\
i+1
\end{array}\right)
$$

and the product rule for differentiation, to obtain 


$$
\begin{aligned}
D_{c, h}\left(D_{s}^{k} \log \left|c^{\prime}\right|\right) & =D_{s}^{k}\left\langle D_{s} h, v\right\rangle-\sum_{j=0}^{k-1} \sum_{i=0}^{j}\left(\begin{array}{l}
j \\
i
\end{array}\right)\left(D_{s}^{k-j+j-i} \log \left|c^{\prime}\right|\right) D_{s}^{i}\left\langle D_{s} h, v\right\rangle \\
& =D_{s}^{k}\left\langle D_{s} h, v\right\rangle-\sum_{i=0}^{k-1} \sum_{j=i}^{k-1}\left(\begin{array}{l}
j \\
i
\end{array}\right)\left(D_{s}^{k-i} \log \left|c^{\prime}\right|\right) D_{s}^{i}\left\langle D_{s} h, v\right\rangle \\
& =D_{s}^{k}\left\langle D_{s} h, v\right\rangle-\sum_{i=0}^{k-1}\left(\begin{array}{c}
k \\
i+1
\end{array}\right)\left(D_{s}^{k-i} \log \left|c^{\prime}\right|\right) D_{s}^{i}\left\langle D_{s} h, v\right\rangle,
\end{aligned}
$$

which completes the first part of the proof. Along the same lines, we also get the variation of $D_{s}^{k} \alpha$.

THEOREM 4.7. Assume that the metric $G$ on $\operatorname{Imm}\left(S^{1}, \mathbb{R}^{2}\right)$ satisfies

$$
\int_{S^{1}}|h|^{2}+\left|D_{s}^{n} h\right|^{2} \mathrm{~d} s \leqslant A G_{c}(h, h)
$$

for some $n \geqslant 2$ and some $A>0$. For each $c_{0} \in \operatorname{Imm}\left(S^{1}, \mathbb{R}^{2}\right)$ and $N>0$ there exists a constant $C=C\left(c_{0}, N\right)$ such that, for all $c_{1}, c_{2} \in \operatorname{Imm}\left(S^{1}, \mathbb{R}^{2}\right)$ with $\operatorname{dist}^{G}\left(c_{0}, c_{i}\right)<N$ and all $0 \leqslant k \leqslant n-2$, we have

$$
\begin{gathered}
\left\|\left(D_{c_{1}}^{k} \kappa_{1}\right) \sqrt{\left|c_{1}^{\prime}\right|}-\left(D_{c_{2}}^{k} \kappa_{2}\right) \sqrt{\left|c_{2}^{\prime}\right|}\right\|_{L^{2}(d \theta)} \leqslant C \operatorname{dist}^{G}\left(c_{1}, c_{2}\right) \\
\left\|\left(D_{c_{1}}^{k+1} \log \left|c_{1}^{\prime}\right|\right) \sqrt{\left|c_{1}^{\prime}\right|}-\left(D_{c_{2}}^{k+1} \log \left|c_{2}^{\prime}\right|\right) \sqrt{\left|c_{2}^{\prime}\right|}\right\|_{L^{2}(d \theta)} \leqslant C \operatorname{dist}^{G}\left(c_{1}, c_{2}\right) .
\end{gathered}
$$

In particular, the functions

$$
\begin{array}{r}
\left(D_{s}^{k} \kappa\right) \sqrt{\left|c^{\prime}\right|}:\left(\operatorname{Imm}\left(S^{1}, \mathbb{R}^{2}\right), \operatorname{dist}^{G}\right) \rightarrow L^{2}\left(S^{1}, \mathbb{R}\right) \\
\left(D_{s}^{k+1} \log \left|c^{\prime}\right|\right) \sqrt{\left|c^{\prime}\right|}:\left(\operatorname{Imm}\left(S^{1}, \mathbb{R}^{2}\right), \operatorname{dist}^{G}\right) \rightarrow L^{2}\left(S^{1}, \mathbb{R}\right)
\end{array}
$$

are continuous and Lipschitz continuous on every metric ball.

Proof. We have $\operatorname{dist}^{G}\left(c_{1}, c_{2}\right)<2 N$ by the triangle inequality. Let $c(t, \theta)$ be a path between $c_{1}$ and $c_{2}$ with $\operatorname{Len}^{G}(c) \leqslant 3 N$. Then

$$
\begin{aligned}
\operatorname{dist}^{G}\left(c_{0}, c(t)\right) & \leqslant \operatorname{dist}^{G}\left(c_{0}, c_{1}\right)+\operatorname{dist}^{G}\left(c_{1}, c(t)\right) \\
& \leqslant N+\operatorname{Len}^{G}\left(\left.c\right|_{[0, t]}\right) \\
& \leqslant N+3 N \leqslant 4 N ;
\end{aligned}
$$

thus any path of this kind remains within a ball of radius $4 N$ around $c_{0}$. 
We will prove the theorem for each $n$ by induction over $k$. The proof of the continuity of $\left(D_{s}^{k} \kappa\right) \sqrt{\left|c^{\prime}\right|}$ does not depend on the continuity of $\left(D_{s}^{k+1} \log \left|c^{\prime}\right|\right) \sqrt{\left|c^{\prime}\right|}$. Thus, even if we prove both statements in parallel, we will assume that we have established the continuity and local Lipschitz continuity of $\left(D_{s}^{k} \kappa\right) \sqrt{\left|c^{\prime}\right|}$ when estimating $\left\|\partial_{t}\left(\left(D_{s}^{k+1} \log \left|c^{\prime}\right|\right) \sqrt{\left|c^{\prime}\right|}\right)\right\|_{L^{2}(d \theta)}$ below; in particular, we will need that

$$
\left\|D_{S}^{k} \kappa\right\|_{L^{2}(d s)} \quad \text { remains bounded along the path. }
$$

The proof consists of two steps. First, we show that the following estimates hold along $c(t, \theta)$ :

$$
\begin{aligned}
& \left\|\partial_{t}\left(\left(D_{s}^{k} \kappa\right) \sqrt{\left|c^{\prime}\right|}\right)\right\|_{L^{2}(d \theta)} \lesssim c_{0}, N\left(1+\left\|D_{s}^{k} \kappa\right\|_{L^{2}(d s)}\right) \sqrt{G_{c}\left(c_{t}, c_{t}\right)} \\
& \left\|\partial_{t}\left(\left(D_{s}^{k+1} \log \left|c^{\prime}\right|\right) \sqrt{\left|c^{\prime}\right|}\right)\right\|_{L^{2}(d \theta)} \lesssim_{c_{0}, N}\left(1+\left\|D_{s}^{k+1} \log \left|c^{\prime}\right|\right\|_{L^{2}(d s)}\right) \sqrt{G_{c}\left(c_{t}, c_{t}\right)} .
\end{aligned}
$$

Then we apply Gronwall's inequality to prove the theorem.

Step 1. For $k=0$, we have

$$
\begin{aligned}
& \partial_{t}\left(\kappa \sqrt{\left|c^{\prime}\right|}\right)=\left\langle D_{s}^{2} c_{t}, n\right\rangle \sqrt{\left|c^{\prime}\right|}-\frac{3}{2} \kappa\left\langle D_{s} c_{t}, v\right\rangle \sqrt{\left|c^{\prime}\right|} \\
& \partial_{t}\left(\left(D_{s} \log \left|c^{\prime}\right|\right) \sqrt{\left|c^{\prime}\right|}\right)=\left\langle D_{s}^{2} c_{t}, v\right\rangle \sqrt{\left|c^{\prime}\right|}+\kappa\left\langle D_{s} c_{t}, n\right\rangle \sqrt{\left|c^{\prime}\right|}- \\
&-\frac{1}{2}\left(D_{s} \log \left|c^{\prime}\right|\right)\left\langle D_{s} c_{t}, v\right\rangle \sqrt{\left|c^{\prime}\right|},
\end{aligned}
$$

and therefore

$$
\begin{array}{r}
\left\|\partial_{t}\left(\kappa \sqrt{\left|c^{\prime}\right|}\right)\right\|_{L^{2}(d \theta)} \leqslant\left\|D_{s}^{2} c_{t}\right\|_{L^{2}(d s)}+\frac{3}{2}\|\kappa\|_{L^{2}(d s)}\left\|D_{s} c_{t}\right\|_{L^{\infty}} \\
\left\|\partial_{t}\left(\left(D_{s} \log \left|c^{\prime}\right|\right) \sqrt{\left|c^{\prime}\right|}\right)\right\|_{L^{2}(d \theta)} \leqslant\left\|D_{s}^{2} c_{t}\right\|_{L^{2}(d s)}+\|\kappa\|_{L^{2}(d s)}\left\|D_{s} c_{t}\right\|_{L^{\infty}} \\
+\frac{1}{2}\left\|D_{s} \log \left|c^{\prime}\right|\right\|_{L^{2}(d s)}\left\|D_{s} c_{t}\right\|_{L^{\infty}} .
\end{array}
$$

Note that the length $\ell_{c}$ is bounded along $c(t, \theta)$ by Corollary 4.2. Using the Poincaré inequalities from Lemma 2.14, and assumption (9), we obtain

$$
\begin{aligned}
& \left\|\partial_{t}\left(\kappa \sqrt{\left|c^{\prime}\right|}\right)\right\|_{L^{2}(d \theta)} \lesssim c_{0}, N\left(1+\|\kappa\|_{L^{2}(d s)}\right) \sqrt{G_{c}\left(c_{t}, c_{t}\right)} \\
& \left\|\partial_{t}\left(\left(D_{s} \log \left|c^{\prime}\right|\right) \sqrt{\left|c^{\prime}\right|}\right)\right\|_{L^{2}(d \theta)} \lesssim c_{0}, N\left(1+\left\|D_{s} \log \left|c^{\prime}\right|\right\|_{L^{2}(d s)}\right) \sqrt{G_{c}\left(c_{t}, c_{t}\right)} .
\end{aligned}
$$


For the second estimate, we used the boundedness of $\|\kappa\|_{L^{2}(d s)}$ from (10). This concludes the proof of step 1 for $k=0$.

Now consider $k>0$, and assume that the theorem has been shown for $k-1$. Along $c(t, \theta)$, the following objects are bounded.

- $\ell_{c}$ by Corollary 4.2, allowing us to use Poincaré inequalities;

- $\left\|D_{s}^{k-1} \kappa\right\|_{L^{2}(d s)}$ and $\left\|D_{s}^{k} \log \left|c^{\prime}\right|\right\|_{L^{2}(d s)}$ by induction; and

- $\left\|D_{s}^{j} \kappa\right\|_{L^{\infty}}$ and $\left\|D_{s}^{j+1} \log \left|c^{\prime}\right|\right\|_{L^{\infty}}$ for $0 \leqslant j \leqslant k-2$ via Poincaré inequalities.

We also have the following bounds, which are valid for both $v$ and $n$.

- $\left\|D_{s}^{j}\left\langle D_{s} c_{t}, v\right\rangle\right\|_{L^{2}(d s)} \lesssim_{c_{0}, N} \sqrt{G_{c}\left(c_{t}, c_{t}\right)}$ for $0 \leqslant j \leqslant k$.

This is clear for $j \leqslant k-1$, since the highest derivative of $\kappa$ that appears due to the Frenet equations is $D_{s}^{k-2} \kappa$, and thus all terms involving $\kappa$ can be bounded by the $L^{\infty}$-norm. For $j=k$, we have

$$
D_{s}^{k}\left\langle D_{s} c_{t}, v\right\rangle=\left\langle D_{s} c_{t}, D_{s}^{k} v\right\rangle+\sum_{j=1}^{k}\left(\begin{array}{l}
k \\
j
\end{array}\right)\left\langle D_{s}^{j+1} c_{t}, D_{s}^{k-j} v\right\rangle
$$

and

$$
D_{s}^{k} v=\left(D_{s}^{k-1} \kappa\right) n+\text { lower-order derivatives in } \kappa .
$$

Thus

$$
\left\|D_{s}^{k} v\right\|_{L^{2}(d s)} \leqslant\left\|D_{s}^{k-1} \kappa\right\|_{L^{2}(d s)}+\cdots \lesssim_{c_{0}, N} 1
$$

Hence we get

$$
\begin{aligned}
\left\|D_{s}^{k}\left\langle D_{s} c_{t}, v\right\rangle\right\|_{L^{2}(d s)} \leqslant & \left\|D_{s} c_{t}\right\|_{L^{\infty}}\left\|D_{s}^{k} v\right\|_{L^{2}(d s)} \\
& +\sum_{j=1}^{k}\left(\begin{array}{c}
k \\
j
\end{array}\right)\left\|D_{s}^{j+1} c_{t}\right\|_{L^{2}(d s)}\left\|D_{s}^{k-j} v\right\|_{L^{\infty}} \\
& \lesssim_{c_{0}, N} \sqrt{G_{c}\left(c_{t}, c_{t}\right)} .
\end{aligned}
$$

- $\left\|D_{s}^{k+1}\left\langle D_{s} c_{t}, v\right\rangle\right\|_{L^{2}(d s)} \lesssim_{c_{0}, N}\left(1+\left\|D_{s}^{k} \kappa\right\|_{L^{2}(d s)}\right) \sqrt{G_{c}\left(c_{t}, c_{t}\right)}$.

We obtain this bound from

$$
\begin{aligned}
D_{s}^{k+1}\left\langle D_{s} c_{t}, v\right\rangle= & \left\langle D_{s}^{k+2} c_{t}, v\right\rangle+\left\langle D_{s} c_{t}, D_{s}^{k+1} v\right\rangle \\
& +\sum_{j=1}^{k}\left(\begin{array}{c}
k+1 \\
j
\end{array}\right)\left\langle D_{s}^{k+2-j} c_{t}, D_{s}^{j} v\right\rangle .
\end{aligned}
$$


Taking the $L^{2}(d s)$-norm, we get

$$
\begin{aligned}
& \left\|D_{s}^{k+1}\left\langle D_{s} c_{t}, v\right\rangle\right\|_{L^{2}(d s)} \\
& \leqslant\left\|D_{s}^{k+2} c_{t}\right\|_{L^{2}(d s)}+\left\|D_{s} c_{t}\right\|_{L^{\infty}}\left\|D_{s}^{k+1} v\right\|_{L^{2}(d s)} \\
& \quad+\sum_{j=1}^{k}\left(\begin{array}{c}
k+1 \\
j
\end{array}\right)\left\|D_{s}^{k+2-j} c_{t}\right\|_{L^{2}(d s)}\left\|D_{s}^{j} v\right\|_{L^{\infty}} \\
& \quad \lesssim c_{0}, N \\
& \sqrt{G_{c}\left(c_{t}, c_{t}\right)}+\left(1+\left\|D_{s}^{k} \kappa\right\|_{L^{2}(d s)}\right) \sqrt{G_{c}\left(c_{t}, c_{t}\right)}+\sqrt{G_{c}\left(c_{t}, c_{t}\right)},
\end{aligned}
$$

thus showing the claim.

Equation (8) from Lemma 4.6, rewritten for $\kappa$, is

$$
D_{c, h}\left(D_{s}^{k} \kappa\right)=D_{s}^{k+1}\left\langle D_{s} h, n\right\rangle-\sum_{j=0}^{k}\left(\begin{array}{c}
k+1 \\
j+1
\end{array}\right)\left(D_{s}^{k-j} \kappa\right) D_{s}^{j}\left\langle D_{s} h, v\right\rangle .
$$

Thus we get

$$
\begin{gathered}
\partial_{t}\left(\left(D_{s}^{k} \kappa\right) \sqrt{\left|c^{\prime}\right|}\right)=\left(D_{s}^{k+1}\left\langle D_{s} c_{t}, n\right\rangle\right) \sqrt{\left|c^{\prime}\right|}-\left(k+\frac{1}{2}\right)\left(D_{s}^{k} \kappa\right)\left\langle D_{s} c_{t}, v\right\rangle \sqrt{\left|c^{\prime}\right|} \\
-\left(\begin{array}{c}
k+1 \\
2
\end{array}\right)\left(D_{s}^{k-1} \kappa\right)\left(D_{s}\left\langle D_{s} c_{t}, v\right\rangle\right) \sqrt{\left|c^{\prime}\right|}-\sum_{j=2}^{k}\left(\begin{array}{c}
k+1 \\
j+1
\end{array}\right)\left(D_{s}^{k-j} \kappa\right) D_{s}^{j}\left\langle D_{s} c_{t}, v\right\rangle \sqrt{\left|c^{\prime}\right|},
\end{gathered}
$$

and hence, by taking norms,

$$
\begin{aligned}
&\left\|\partial_{t}\left(\left(D_{s}^{k} \kappa\right) \sqrt{\left|c^{\prime}\right|}\right)\right\|_{L^{2}(d \theta)} \leqslant\left\|D_{s}^{k+1}\left\langle D_{s} c_{t}, n\right\rangle\right\|_{L^{2}(d s)} \\
&+\left(k+\frac{1}{2}\right)\left\|D_{s}^{k} \kappa\right\|_{L^{2}(d s)}\left\|\left\langle D_{s} c_{t}, v\right\rangle\right\|_{L^{\infty}} \\
&+\left(\begin{array}{c}
k+1 \\
2
\end{array}\right)\left\|D_{s}^{k-1} \kappa\right\|_{L^{2}(d s)}\left\|D_{s}\left\langle D_{s} c_{t}, v\right\rangle\right\|_{L^{\infty}} \\
&+\sum_{j=2}^{k}\left(\begin{array}{c}
k+1 \\
j+1
\end{array}\right)\left\|D_{s}^{k-j} \kappa\right\|_{L^{\infty}}\left\|D_{s}^{j}\left\langle D_{s} c_{t}, v\right\rangle\right\|_{L^{2}(d s)} \\
& \lesssim_{c_{0}, N}\left(1+\left\|D_{s}^{k} \kappa\right\|_{L^{2}(d s)}\right) \sqrt{G_{c}\left(c_{t}, c_{t}\right)} .
\end{aligned}
$$

For $\left(D_{s}^{k+1} \log \left|c^{\prime}\right|\right) \sqrt{\left|c^{\prime}\right|}$, we proceed similarly. The time derivative is $\partial_{t}\left(\left(D_{s}^{k+1} \log \left|c^{\prime}\right|\right) \sqrt{\left|c^{\prime}\right|}\right)=D_{s}^{k+1}\left\langle D_{s} c_{t}, v\right\rangle \sqrt{\left|c^{\prime}\right|}$

$$
-\left(k+\frac{1}{2}\right)\left(D_{s}^{k+1} \log \left|c^{\prime}\right|\right)\left\langle D_{s} c_{t}, v\right\rangle \sqrt{\left|c^{\prime}\right|}
$$




$$
\begin{aligned}
& -\left(\begin{array}{c}
k+1 \\
2
\end{array}\right)\left(D_{s}^{k} \log \left|c^{\prime}\right|\right) D_{s}\left\langle D_{s} c_{t}, v\right\rangle \sqrt{\left|c^{\prime}\right|} \\
& -\sum_{j=2}^{k}\left(\begin{array}{c}
k+1 \\
j+1
\end{array}\right)\left(D_{s}^{k+1-j} \log \left|c^{\prime}\right|\right) D_{s}^{j}\left\langle D_{s} c_{t}, v\right\rangle \sqrt{\left|c^{\prime}\right|}
\end{aligned}
$$

which can be estimated by

$$
\begin{aligned}
& \left\|\partial_{t}\left(\left(D_{s}^{k+1} \log \left|c^{\prime}\right|\right) \sqrt{\left|c^{\prime}\right|}\right)\right\|_{L^{2}(d \theta)} \\
& \leqslant\left\|D_{s}^{k+1}\left\langle D_{s} c_{t}, v\right\rangle\right\|_{L^{2}(d s)}+\left(k+\frac{1}{2}\right)\left\|D_{s}^{k+1} \log \left|c^{\prime}\right|\right\|_{L^{2}(d s)}\left\|D_{s} c_{t}\right\|_{L^{\infty}} \\
& \quad+\left(\begin{array}{c}
k+1 \\
2
\end{array}\right)\left\|D_{s}^{k} \log \left|c^{\prime}\right|\right\|_{L^{2}(d s)}\left\|D_{s}\left\langle D_{s} c_{t}, v\right\rangle\right\|_{L^{\infty}} \\
& \quad+\sum_{j=2}^{k}\left(\begin{array}{c}
k+1 \\
j+1
\end{array}\right)\left\|D_{s}^{k+1-j} \log \left|c^{\prime}\right|\right\|_{L^{\infty}}\left\|D_{s}^{j}\left\langle D_{s} c_{t}, v\right\rangle\right\|_{L^{2}(d s)} \\
& \lesssim_{c_{0}, N}\left(1+\left\|D_{s}^{k+1} \log \left|c^{\prime}\right|\right\|_{L^{2}(d s)}\right) \sqrt{G_{c}\left(c_{t}, c_{t}\right)} .
\end{aligned}
$$

Step 2. The proof of this step depends only on the estimates (11) and (12). We have a path $c(t, \theta)$ between $c_{1}$ and $c_{2}$. We write again $D_{c_{1}}$ and $D_{c(t)}$ for $D_{s_{c_{1}}}$ and $D_{s_{c(t)}}$, respectively. Define the functions

$$
\begin{aligned}
& A(t)=\left\|\left(D_{c_{1}}^{k} \kappa_{1}\right) \sqrt{\left|c_{1}^{\prime}\right|}-\left(D_{c(t)}^{k} \kappa(t)\right) \sqrt{\left|c(t)^{\prime}\right|}\right\|_{L^{2}(d \theta)} \\
& B(t)=\left\|\left(D_{c_{1}}^{k+1} \log \left|c_{1}^{\prime}\right|\right) \sqrt{\left|c_{1}^{\prime}\right|}-\left(D_{c(t)}^{k+1} \log \left|c(t)^{\prime}\right|\right) \sqrt{\left|c(t)^{\prime}\right|}\right\|_{L^{2}(d \theta)} .
\end{aligned}
$$

From

$$
\left.\left(D_{c}^{k} \kappa\right) \sqrt{\left|c^{\prime}\right|}(t, \theta)-\left(D_{c_{1}}^{k} \kappa_{1}\right) \sqrt{\left|c_{1}^{\prime}\right|}(\theta)=\int_{0}^{t} \partial_{t}\left(D_{s}^{k} \kappa\right) \sqrt{\left|c^{\prime}\right|}\right)(\tau, \theta) d \tau
$$

we get, by taking norms,

$$
\begin{aligned}
A(t) & \leqslant \int_{0}^{t}\left\|\partial_{t}\left(D_{s}^{k} \kappa \sqrt{\left|c^{\prime}\right|}\right)\right\|_{L^{2}(d \theta)} d \tau \\
& \lesssim c_{0}, N \int_{0}^{t}\left(1+\left\|D_{s}^{k} \kappa\right\|_{L^{2}(d s)}\right) \sqrt{G_{c}\left(c_{t}, c_{t}\right)} d \tau \\
& \lesssim c_{0}, N \int_{0}^{t}\left(1+\left\|D_{s}^{k} \kappa_{1}\right\|_{L^{2}(d s)}+A(\tau)\right) \sqrt{G_{c}\left(c_{t}, c_{t}\right)} d \tau
\end{aligned}
$$


Now we use Gronwall's inequality, Corollary 2.11, to obtain

$$
A(t) \lesssim_{c_{0}, N}\left(1+\left\|D_{s}^{k} \kappa_{1}\right\|_{L^{2}(d s)}\right) \int_{0}^{t} \sqrt{G_{c}\left(c_{t}, c_{t}\right)} d \tau .
$$

Taking the infimum over all paths and evaluating at $t=1$ then yields the following inequality, which is almost the desired one.

$$
\left\|\left(D_{c_{1}}^{k} \kappa_{1}\right) \sqrt{\left|c_{1}^{\prime}\right|}-\left(D_{c_{2}}^{k} \kappa_{2}\right) \sqrt{\left|c_{2}^{\prime}\right|}\right\|_{L^{2}(d \theta)} \lesssim c_{0}, N\left(1+\left\|D_{s}^{k} \kappa_{1}\right\|_{L^{2}(d s)}\right) \operatorname{dist}^{G}\left(c_{1}, c_{2}\right) .
$$

To bound $\left\|D_{s}^{k} \kappa_{1}\right\|_{L^{2}(d s)}$, which appears on the right-hand side, we apply (15) with $c_{2}=c_{0}$.

$$
\begin{aligned}
\left\|D_{s}^{k} \kappa_{1}\right\|_{L^{2}(d s)} & \leqslant\left\|D_{c_{1}}^{k}\left(\kappa_{1}\right) \sqrt{\left|c_{1}^{\prime}\right|}-D_{c_{0}}^{k}\left(\kappa_{0}\right) \sqrt{\left|c_{0}^{\prime}\right|}\right\|_{L^{2}(d \theta)}+\left\|D_{s}^{k} \kappa_{0}\right\|_{L^{2}(d s)} \\
& \lesssim c_{0, N}\left(1+\left\|D_{s}^{k} \kappa_{0}\right\|_{L^{2}(d s)}\right) \operatorname{dist}^{G}\left(c_{0}, c_{1}\right)+\left\|D_{s}^{k} \kappa_{0}\right\|_{L^{2}(d s)} \lesssim_{c_{0}, N} 1 .
\end{aligned}
$$

This concludes the proof for $\left(D_{s}^{k} \kappa\right) \sqrt{\left|c^{\prime}\right|}$. For $\left(D_{s}^{k+1} \log \left|c^{\prime}\right|\right) \sqrt{\left|c^{\prime}\right|}$, we proceed in the same way with $B(t)$ in place of $A(t)$ using the estimate (12).

REMARK 4.8. Theorem 4.7 makes no statement about the continuity or local Lipschitz continuity of the function $\log \left|c^{\prime}\right| \sqrt{\left|c^{\prime}\right|}$ when $G$ is a Sobolev metric of order 1 . In fact it appears that one needs a metric of order $n \geqslant 2$. In that case, one can use the variational formula,

$$
D_{c, h}\left(\log \left|c^{\prime}\right| \sqrt{\left|c^{\prime}\right|}\right)=\left(1+\frac{1}{2} \log \left|c^{\prime}\right|\right)\left\langle D_{s} h, v\right\rangle \sqrt{\left|c^{\prime}\right|},
$$

and the same method of proof (with $n \geqslant 2$ one can estimate $\left\langle D_{s} h, v\right\rangle$ using the $L^{\infty}$-norm) to show that

$$
\left(\log \left|c^{\prime}\right|\right) \sqrt{\left|c^{\prime}\right|}:\left(\operatorname{Imm}\left(S^{1}, \mathbb{R}^{2}\right), \operatorname{dist}^{G}\right) \rightarrow L^{2}\left(S^{1}, \mathbb{R}^{2}\right)
$$

is continuous and Lipschitz continuous on every metric ball.

REMARK 4.9. In a similar way, we can also obtain continuity in $L^{\infty}$ instead of $L^{2}$. Assume that the metric satisfies (9) with $n \geqslant 3$. Then for all $1 \leqslant k \leqslant n-2$ the functions

$$
\begin{aligned}
D_{s}^{k-1} \kappa:\left(\operatorname{Imm}\left(S^{1}, \mathbb{R}^{2}\right), \operatorname{dist}^{G}\right) & \rightarrow L^{\infty}\left(S^{1}, \mathbb{R}\right) \\
D_{s}^{k} \log \left|c^{\prime}\right|:\left(\operatorname{Imm}\left(S^{1}, \mathbb{R}^{2}\right), \operatorname{dist}^{G}\right) & \rightarrow L^{\infty}\left(S^{1}, \mathbb{R}\right)
\end{aligned}
$$


are continuous and Lipschitz continuous on every metric ball. To prove this, we follow the proof of Theorem 4.7, and replace the estimates (11) and (12) with

$$
\begin{aligned}
&\left\|\partial_{t}\left(D_{s}^{k-1} \kappa\right)\right\|_{L^{\infty}} \lesssim c_{0}, N \\
&\left\|\partial_{t}\left(D_{s}^{k} \log \left|c^{\prime}\right|\right)\right\|_{L^{\infty}} \lesssim c_{0}, N \\
&\left(1+\left\|D_{s}^{k-1} \kappa\right\|_{L^{\infty}}\right) \sqrt{G_{c}\left(c_{t}, c_{t}\right)} \\
&\left.\log \left|c^{\prime}\right| \|_{L^{\infty}}\right) \sqrt{G_{c}\left(c_{t}, c_{t}\right)},
\end{aligned}
$$

which can be established in the same way.

We also have $L^{\infty}$-continuity of $\log \left|c^{\prime}\right|$, when $n=2$. Since we will use it in the proof of geodesic completeness, we shall provide an explicit proof in Lemma 4.10.

LEMMA 4.10. Let the metric $G$ on $\operatorname{Imm}\left(S^{1}, \mathbb{R}^{2}\right)$ satisfy

$$
\int_{S^{1}}|h|^{2}+\left|D_{s}^{n} h\right|^{2} d s \leqslant A G_{c}(h, h)
$$

for some $n \geqslant 2$ and some $A>0$. Given $c_{0} \in \operatorname{Imm}\left(S^{1}, \mathbb{R}^{2}\right)$ and $N>0$, there exists a constant $C=C\left(c_{0}, N\right)$ such that, for all $c_{1}, c_{2} \in \operatorname{Imm}\left(S^{1}, \mathbb{R}^{2}\right)$ with $\operatorname{dist}^{G}\left(c_{0}\right.$, $\left.c_{i}\right)<N$, we have

$$
\left\|\log \left|c_{1}^{\prime}\right|-\log \left|c_{2}^{\prime}\right|\right\|_{L^{\infty}} \leqslant C \operatorname{dist}^{G}\left(c_{1}, c_{2}\right) .
$$

In particular, the function

$$
\log \left|c^{\prime}\right|:\left(\operatorname{Imm}\left(S^{1}, \mathbb{R}^{2}\right), \operatorname{dist}^{G}\right) \rightarrow L^{\infty}\left(S^{1}, \mathbb{R}\right)
$$

is continuous and Lipschitz continuous on every metric ball.

Proof. Fix $\theta \in S^{1}$ and $c_{1} \in \operatorname{Imm}\left(S^{1}, \mathbb{R}^{2}\right)$ satisfying $\operatorname{dist}^{G}\left(c_{0}, c_{1}\right)<N$, and let $c(t, \theta)$ be a path between $c_{0}$ and $c_{1}$ with $\operatorname{Len}^{G}(c) \leqslant 2 N$. Then

$$
\partial_{t}\left(\log \left|c^{\prime}(\theta)\right|\right)=\left\langle D_{s} c_{t}(\theta), v(\theta)\right\rangle .
$$

After integrating and taking norms, we get

$$
|\log | c_{1}^{\prime}(\theta)|-\log | c_{0}^{\prime}(\theta)|| \leqslant \int_{0}^{1}\left|D_{s} c_{t}(t, \theta)\right| \mathrm{d} t .
$$

Using Poincaré inequalities and Corollary 4.2, we can estimate

$$
\begin{aligned}
\left|D_{s} c_{t}(\theta)\right| \leqslant & \frac{\sqrt{\ell_{c}}}{2}\left\|D_{s}^{2} c_{t}\right\|_{L^{2}(d s)} \\
& \leqslant \frac{\sqrt{\ell_{c}}}{2} \sqrt{\left\|c_{t}\right\|_{L^{2}(d s)}^{2}+\left\|D_{s}^{n} c_{t}\right\|_{L^{2}(d s)}^{2}} \leqslant \frac{1}{2} \sqrt{\ell_{c} A} \sqrt{G_{c}\left(c_{t}, c_{t}\right)} .
\end{aligned}
$$


Thus, by taking the infimum over all paths between $c_{0}$ and $c_{1}$, we get

$$
\left\|\log \left|c_{1}^{\prime}\right|-\log \left|c_{0}^{\prime}\right|\right\|_{L^{\infty}} \lesssim c_{0}, N \operatorname{dist}^{G}\left(c_{0}, c_{1}\right) .
$$

REMARK. An explicit value for the constant is given by

$$
C\left(c_{0}, N\right)=\frac{1}{2} \sqrt{A}\left(\sqrt{\ell_{c_{0}}}+\frac{1}{2} \sqrt{A} N\right) .
$$

This can be found by combining the estimates (16) and (6).

This corollary gives us upper and lower bounds on $\left|c^{\prime}(\theta)\right|$ in terms of the geodesic distance. Therefore, a geodesic $c(t, \theta)$ for a Sobolev metric with order at least 2 cannot leave $\operatorname{Imm}\left(S^{1}, \mathbb{R}^{2}\right)$ by having $c^{\prime}(t, \theta)=0$ for some $(t, \theta)$.

COROLlaRY 4.11. Under the assumptions of Lemma 4.10, given $c_{0} \in \operatorname{Imm}\left(S^{1}\right.$, $\left.\mathbb{R}^{2}\right)$ and $N>0$, there exists a constant $C=C\left(c_{0}, N\right)$ such that

$$
\left\|c^{\prime}\right\|_{L^{\infty}} \leqslant C \text { and }\left\|\frac{1}{\left|c^{\prime}\right|}\right\|_{L^{\infty}} \leqslant C
$$

hold for all $c \in \operatorname{Imm}\left(S^{1}, \mathbb{R}^{2}\right)$ with $\operatorname{dist}^{G}\left(c_{0}, c\right)<N$.

Proof. By Lemma 4.10, we have

$$
\log \left|c^{\prime}(\theta)\right| \leqslant\left\|\log \left|c_{0}^{\prime}\right|\right\|_{L^{\infty}}+\left\|\log \left|c^{\prime}\right|-\log \left|c_{0}^{\prime}\right|\right\|_{L^{\infty}} \lesssim_{c_{0}, N} 1 .
$$

Now apply exp and take the supremum over $\theta$ to obtain $\left\|c^{\prime}\right\|_{L^{\infty}} \lesssim_{c_{0}, N} 1$. Similarly, by starting from

$$
-\log \left|c^{\prime}(\theta)\right| \leqslant\left\|\log \left|c_{0}^{\prime}\right|\right\|_{L^{\infty}}+\left\|\log \left|c^{\prime}\right|-\log \left|c_{0}^{\prime}\right|\right\|_{L^{\infty}} \lesssim c_{0}, N 1 .
$$

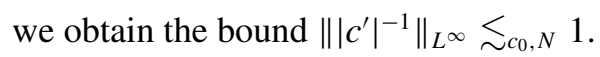

REMARK. Using the explicit constant for Lemma 4.10, we can obtain the following more explicit inequalities for Corollary 4.11,

$$
\begin{aligned}
\left|c^{\prime}(\theta)\right| & \leqslant\left|c_{0}^{\prime}(\theta)\right| \exp \left(\frac{1}{2} \sqrt{A} N\left(\sqrt{\ell_{c_{0}}}+\frac{1}{2} \sqrt{A} N\right)\right) \\
\left|c^{\prime}(\theta)\right|^{-1} & \leqslant\left|c_{0}^{\prime}(\theta)\right|^{-1} \exp \left(\frac{1}{2} \sqrt{A} N\left(\sqrt{\ell_{c_{0}}}+\frac{1}{2} \sqrt{A} N\right)\right) .
\end{aligned}
$$

REMARK 4.12. To simplify the exposition, the results in this section were formulated on the space $\operatorname{Imm}\left(S^{1}, \mathbb{R}^{2}\right)$ of smooth immersions. If $G$ is a Sobolev metric of order $n$ with $n \geqslant 2$, we can replace $\operatorname{Imm}\left(S^{1}, \mathbb{R}^{2}\right)$ by $\operatorname{Imm}^{n}\left(S^{1}, \mathbb{R}^{2}\right)$ in all statements of this section with the same proofs. 


\section{Geodesic completeness for Sobolev metrics}

On the space $H^{n}\left(S^{1}, \mathbb{R}^{d}\right)$ we have two norms: the $H^{n}(d \theta)$-norm as well as the $H^{n}(d s)$-norm, which depends on the choice of a curve $c \in \operatorname{Imm}\left(S^{1}, \mathbb{R}^{2}\right)$. Although the norms are equivalent, the constant in the inequality

$$
C^{-1}\|h\|_{H^{k}(d \theta)} \leqslant\|h\|_{H^{k}(d s)} \leqslant C\|h\|_{H^{k}(d \theta)},
$$

depends in general on the curve and its derivatives. The next lemma shows that, if $c$ remains in a metric ball with respect to the geodesic distance, then the constant depends only on the center and the radius of the ball.

LEMMA 5.1. Let the metric $G$ on $\operatorname{Imm}\left(S^{1}, \mathbb{R}^{2}\right)$ satisfy

$$
\int_{S^{1}}|h|^{2}+\left|D_{s}^{n} h\right|^{2} d s \leqslant A G_{c}(h, h)
$$

for some $n \geqslant 2$ and some $A>0$. Given $c_{0} \in \operatorname{Imm}\left(S^{1}, \mathbb{R}^{2}\right)$ and $N>0$, there exists a constant $C=C\left(c_{0}, N\right)$ such that, for $0 \leqslant k \leqslant n$,

$$
C^{-1}\|h\|_{H^{k}(d \theta)} \leqslant\|h\|_{H^{k}(d s)} \leqslant C\|h\|_{H^{k}(d \theta)},
$$

holds for all $c \in \operatorname{Imm}\left(S^{1}, \mathbb{R}^{2}\right)$ with $\mathrm{dist}^{G}\left(c_{0}, c\right)<N$ and all $h \in H^{k}\left(S^{1}, \mathbb{R}^{d}\right)$.

Proof. By definition,

$$
\begin{aligned}
\|u\|_{H^{k}(d \theta)}^{2} & =\|h\|_{L^{2}(d \theta)}^{2}+\left\|\partial_{\theta}^{k} h\right\|_{L^{2}(d \theta)}^{2} \\
\|u\|_{H^{k}(d s)}^{2} & =\|h\|_{L^{2}(d s)}^{2}+\left\|D_{s}^{k} h\right\|_{L^{2}(d s)}^{2} .
\end{aligned}
$$

The estimates

$$
\left(\min _{\theta \in S^{1}}\left|c^{\prime}(\theta)\right|\right)\|h\|_{L^{2}(d \theta)}^{2} \leqslant\|h\|_{L^{2}(d s)}^{2} \leqslant\left\|c^{\prime}\right\|_{L^{\infty}}\|h\|_{L^{2}(d \theta)}^{2},
$$

together with Corollary 4.11 take care of the $L^{2}$-terms. Thus it remains to compare the derivatives $\left\|\partial_{\theta}^{k} h\right\|_{L^{2}(d \theta)}^{2}$ and $\left\|D_{s}^{k} h\right\|_{L^{2}(d s)}^{2}$. From the identities

$$
\begin{aligned}
h^{\prime}= & \left|c^{\prime}\right| D_{s} h \\
h^{\prime \prime}= & \left|c^{\prime}\right|^{2} D_{s}^{2} h+\left(\partial_{\theta}\left|c^{\prime}\right|\right) D_{s} h \\
h^{\prime \prime \prime}= & \left|c^{\prime}\right|^{3} D_{s}^{3} h+3\left|c^{\prime}\right|\left(\partial_{\theta}\left|c^{\prime}\right|\right) D_{s}^{2} h+\left(\partial_{\theta}^{2}\left|c^{\prime}\right|\right) D_{s} h \\
h^{\prime \prime \prime \prime}= & \left|c^{\prime}\right|^{4} D_{s}^{4} h+6\left|c^{\prime}\right|^{2}\left(\partial_{\theta}\left|c^{\prime}\right|\right) D_{s}^{3} h+\left(3\left(\partial_{\theta}\left|c^{\prime}\right|\right)^{2}+4\left|c^{\prime}\right|\left(\partial_{\theta}^{2}\left|c^{\prime}\right|\right)\right) D_{s}^{2} h \\
& +\left(\partial_{\theta}^{3}\left|c^{\prime}\right|\right) D_{s} h,
\end{aligned}
$$


we generalize to

$$
\partial_{\theta}^{k} h=\sum_{j=1}^{k} \sum_{\alpha \in A_{j}} c_{j, \alpha} \prod_{i=0}^{k-1}\left(\partial_{\theta}^{i}\left|c^{\prime}\right|\right)^{\alpha_{i}} D_{s}^{j} h
$$

where $c_{j, \alpha}$ are some constants and $\alpha=\left(\alpha_{0}, \ldots, \alpha_{k-1}\right)$ are multiindices that are summed over the index sets

$$
A_{j}=\left\{\alpha: \sum_{i=0}^{k-1} i \alpha_{i}=k-j, \sum_{i=0}^{k-1} \alpha_{i}=j\right\}
$$

Equation (17) is related to Faà di Bruno's formula [10], and can be proven by induction.

The length $\ell_{c}$ is bounded on the metric ball by Corollary 4.2. Then Lemma 4.7, together with Poincaré inequalities, show that

- $\left\|D_{s}^{n-1} \log \left|c^{\prime}\right|\right\|_{L^{2}(d s)}$ and

- $\left\|D_{s}^{k} \log \left|c^{\prime}\right|\right\|_{L^{\infty}}$ for $1 \leqslant k \leqslant n-2$

are bounded as well. Repeated application of the chain rule for differentiation yields

$$
D_{s}^{k}\left|c^{\prime}\right|=D_{s}^{k}\left(\exp \log \left|c^{\prime}\right|\right)=\left|c^{\prime}\right| D_{s}^{k} \log \left|c^{\prime}\right|+\text { lower } D_{s} \text {-derivatives of } \log \left|c^{\prime}\right| .
$$

Thus $\left\|D_{s}^{n-1}\left|c^{\prime}\right|\right\|_{L^{2}(d s)}$ and $\left\|D_{s}^{k}\left|c^{\prime}\right|\right\|_{L^{\infty}}$ for $1 \leqslant k \leqslant n-2$ are also bounded on metric balls. Next, we apply formula (17) to $h=\left|c^{\prime}\right|$, obtaining

$$
\partial_{\theta}^{k}\left|c^{\prime}\right|=\left|c^{\prime}\right|^{k} D_{s}^{k}\left|c^{\prime}\right|+\text { lower } D_{s} \text {-derivatives of }\left|c^{\prime}\right| \text {. }
$$

Together with Lemma 4.10, this implies that

- $\left\|\partial_{\theta}^{n-1}\left|c^{\prime}\right|\right\|_{L^{2}(d \theta)}$ and

- $\left\|\partial_{\theta}^{k}\left|c^{\prime}\right|\right\|_{L^{\infty}}$ for $0 \leqslant k \leqslant n-2$

are bounded on metric balls.

We proceed by induction over $k$. The case $k=0$ has been dealt with at the beginning of the proof. Assume that $k \leqslant n-1$ and that the equivalence of the norms has been shown for $k-1$. Then the highest derivative of $\left|c^{\prime}\right|$ is $\partial_{\theta}^{k-1}\left|c^{\prime}\right|$, and so in (17) we can estimate every term involving $\left|c^{\prime}\right|$ using the $L^{\infty}$-norm. Thus, using Poincaré inequalities and the equivalence of $L^{2}(d \theta)$ and $L^{2}(d s)$-norms, we get

$$
\left\|\partial_{\theta}^{k} h\right\|_{L^{2}(d \theta)}^{2} \lesssim c_{0}, N\left\|D_{s}^{k} h\right\|_{L^{2}(d s)}^{2} .
$$


For the other inequality, we write

$$
D_{s}^{k} h=\left|c^{\prime}\right|^{-k} \partial_{\theta}^{k} h-\left|c^{\prime}\right|^{-k} \sum_{j=1}^{k-1} \sum_{\alpha \in A_{j}} c_{j, \alpha} \prod_{i=0}^{k-1}\left(\partial_{\theta}^{i}\left|c^{\prime}\right|\right)^{\alpha_{i}} D_{s}^{j} h,
$$

and use the induction assumption $\left\|D_{s}^{j} h\right\|_{L^{2}(d s)}^{2} \lesssim c_{0}, N\left\|\partial_{\theta}^{j} h\right\|_{L^{2}(d \theta)}^{2}$ for $0 \leqslant j<k$.

The only remaining case is $k=n$. There, we have to be a bit more careful, since then $\partial_{\theta}^{n-1}\left|c^{\prime}\right|$ appears in (17), which cannot be bound using the $L^{\infty}$-norm. However, $\partial_{\theta}^{n-1}\left|c^{\prime}\right|$ appears only in the summand $\left(\partial_{\theta}^{n-1}\left|c^{\prime}\right|\right) D_{s} h$; that is, if $\alpha_{n-1} \neq 0$, then $\alpha_{n-1}=1, \alpha_{i}=0$ for $i \neq n-1$ and $\alpha \in A_{1}$. We can estimate this term via

$$
\left\|\left(\partial_{\theta}^{n-1}\left|c^{\prime}\right|\right) D_{s} h\right\|_{L^{2}(d \theta)} \leqslant\left\|\partial_{\theta}^{n-1}\left|c^{\prime}\right|\right\|_{L^{2}(d \theta)}\left\|D_{s} h\right\|_{L^{\infty}},
$$

and then, depending on which inequality we want to show, we can use either of

$$
\begin{aligned}
& \left\|D_{s} h\right\|_{L^{\infty}} \leqslant 2^{-1} \sqrt{\ell_{c}}\left\|D_{s}^{2} h\right\|_{L^{2}(d s)} \\
& \left\|D_{s} h\right\|_{L^{\infty}} \leqslant\left\|\left|c^{\prime}\right|^{-1}\right\|_{L^{\infty}}\left\|\partial_{\theta} h\right\|_{L^{\infty}} \leqslant C\left\|\left|c^{\prime}\right|^{-1}\right\|_{L^{\infty}}\left\|\partial_{\theta}^{2} h\right\|_{L^{2}(d \theta)} .
\end{aligned}
$$

From here, we proceed as for $k<n$.

We saw in Lemma 2.5 that multiplication is a bounded bilinear map on the spaces $H^{k}\left(S^{1}, \mathbb{R}^{d}\right)$ with the $H^{k}(d \theta)$-norm. Since the $H^{k}(d \theta)$-norm and the $H^{k}(d s)$-norm are equivalent, this holds also for the $H^{k}(d s)$-norm. A consequence of Lemma 5.1 is that the constant in the inequality

$$
\|\langle f, g\rangle\|_{H^{k}(d s)} \leqslant C\|f\|_{H^{k}(d s)}\|g\|_{H^{k}(d s)},
$$

again depends only on the center and radius of the geodesic ball.

COROLLARY 5.2. Under the assumptions of Lemma 5.1, there exists a constant $C=C\left(c_{0}, N\right)$ such that, for $c \in \operatorname{Imm}\left(S^{1}, \mathbb{R}^{2}\right)$ with $\operatorname{dist}^{G}\left(c_{0}, c\right)<N$ and $1 \leqslant k$ $\leqslant n$,

$$
\|\langle f, g\rangle\|_{H^{k}(d s)} \leqslant C\|f\|_{H^{k}(d s)}\|g\|_{H^{k}(d s)}
$$

holds for all $f, g \in H^{k}\left(S^{1}, \mathbb{R}^{d}\right)$.

Proof. We use Lemma 5.1 and the boundedness of multiplication on $H^{k}(d \theta)$,

$$
\begin{aligned}
& \|\langle f, g\rangle\|_{H^{k}(d s)} \lesssim c_{0, N}\|\langle f, g\rangle\|_{H^{k}(d \theta)} \\
& \lesssim_{c_{0}, N}\|f\|_{H^{k}(d \theta)}\|g\|_{H^{k}(d \theta)} \lesssim_{c_{0}, N}\|f\|_{H^{k}(d s)}\|g\|_{H^{k}(d s)} .
\end{aligned}
$$


This last lemma shows that the identity

$$
\text { Id }:\left(\operatorname{Imm}^{n}\left(S^{1}, \mathbb{R}^{2}\right), \operatorname{dist}^{G}\right) \rightarrow\left(H^{n}\left(S^{1}, \mathbb{R}^{2}\right), H^{n}(d \theta)\right)
$$

maps bounded sets to bounded sets, and that the same holds for the function

$$
\left(\operatorname{Imm}^{n}\left(S^{1}, \mathbb{R}^{2}\right), \operatorname{dist}^{G}\right) \rightarrow \mathbb{R}, \quad c \mapsto\|c\|_{H^{n}(d s)},
$$

when $G$ is stronger than a Sobolev metric of order $n$.

LEMMA 5.3. Let the metric $G$ on $\operatorname{Imm}\left(S^{1}, \mathbb{R}^{2}\right)$ satisfy

$$
\int_{S^{1}}|h|^{2}+\left|D_{s}^{n} h\right|^{2} d s \leqslant A G_{c}(h, h)
$$

for some $n \geqslant 2$ and some $A>0$. Given $c_{0} \in \operatorname{Imm}\left(S^{1}, \mathbb{R}^{2}\right)$ and $N>0$, there exists a constant $C=C\left(c_{0}, N\right)$ such that

$$
\|c\|_{H^{n}(d \theta)} \leqslant C \quad \text { and } \quad\|c\|_{H^{n}(d s)} \leqslant C .
$$

hold for all $c \in \operatorname{Imm}\left(S^{1}, \mathbb{R}^{2}\right)$ with $\operatorname{dist}^{G}\left(c_{0}, c\right)<N$.

Proof. It is only necessary to prove the boundedness in one of the norms, since Lemma 5.1 will imply the other one. We have

$$
\|c\|_{H^{n}(d s)}^{2}=\|c\|_{L^{2}(d s)}^{2}+\left\|D_{s}^{n} c\right\|_{L^{2}(d s)}^{2}=\|c\|_{L^{2}(d s)}^{2}+\left\|D_{s}^{n-2} \kappa\right\|_{L^{2}(d s)}^{2} .
$$

The boundedness of $\left\|D_{s}^{n-2} \kappa\right\|_{L^{2}(d s)}^{2}$ on metric balls has been shown in Theorem 4.7. For $\|c\|_{L^{2}(d s)}$, we choose a path $c(t)$ from $c_{0}$ to $c=c(1)$ with $\operatorname{Len}^{G}(c(t))<2 N$. Then

$$
\begin{aligned}
\|c\|_{L^{2}(d s)} & \lesssim c_{0}, N \\
& \|c\|_{L^{2}(d \theta)} \leqslant\left\|c-c_{0}\right\|_{L^{2}(d \theta)}+\left\|c_{0}\right\|_{L^{2}(d \theta)} \\
& \lesssim c_{0}, N \\
& \lesssim \int_{0}^{1} \partial_{t} c(t) d t\left\|_{L^{2}(d \theta)} \leqslant \int_{0}^{1}\right\| \partial_{t} c(t) \|_{L^{2}(d \theta)} d t \\
& \int_{0}^{1}\left\|\partial_{t} c(t)\right\|_{L^{2}(d s)} d t \leqslant \operatorname{Len}^{G}(c(t)) \lesssim_{c_{0}, N} 1
\end{aligned}
$$

REMARK 5.4. The proof of Lemma 5.1 shows that under the assumptions of Lemma 5.3 we can choose $C=C\left(c_{0}, N\right)$ such that the additional inequality

$$
\left\|\left|c^{\prime}\right|\right\|_{H^{n-1}(d \theta)} \leqslant C
$$

holds as well. 
Now, we are ready to prove the main theorem.

THEOREM 5.5. Let $n \geqslant 2$, and let $G$ be a Sobolev metric with constant coefficients $a_{i} \geqslant 0$ of order $n$ and $a_{0}, a_{n}>0$. Given $\left(c_{0}, u_{0}\right) \in T \operatorname{Imm}^{n}\left(S^{1}, \mathbb{R}^{2}\right)$, the solution of the geodesic equation for the metric $G$ with initial values $\left(c_{0}, u_{0}\right)$ exists for all time.

COROLlary 5.6. Let the metric $G$ be as in Theorem 5.5. Then the Riemannian manifolds $\left(\operatorname{Imm}^{n}\left(S^{1}, \mathbb{R}^{2}\right), G\right)$ and $\left(\operatorname{Imm}\left(S^{1}, \mathbb{R}^{2}\right), G\right)$ are geodesically complete.

Proof. The geodesic completeness of $\operatorname{Imm}\left(S^{1}, \mathbb{R}^{2}\right)$ follows from Theorem 3.7, since, given smooth initial conditions, the intervals of existence are uniform in the Sobolev order.

Proof of Theorem 5.5. The geodesic equation is equivalent to the following ODE on $\left(T \mathrm{Imm}^{n}\right)^{\prime} \cong \mathrm{Imm}^{n} \times H^{-n}$ :

$$
\begin{aligned}
c_{t} & =\bar{L}_{c}^{-1} p \\
p_{t} & =\frac{1}{2} H_{c}\left(\bar{L}_{c}^{-1} p, \bar{L}_{c}^{-1} p\right),
\end{aligned}
$$

with $p(t)=\bar{L}_{c(t)} u(t)$. Fix initial conditions $(c(0), p(0))$. In order to show that the geodesic with these initial conditions exists for all time, we need to show that, on any finite interval $[0, T)$ on which the geodesic $(c(t), p(t))$ exists, we have that

(A) the closure of $c([0, T))$ in $H^{n}\left(S^{1}, \mathbb{R}^{2}\right)$ is contained in $\operatorname{Imm}^{n}\left(S^{1}, \mathbb{R}^{2}\right)$; and

(B) $\left\|\bar{L}_{c}^{-1} p\right\|_{H^{n}(d \theta)}, \frac{1}{2}\left\|H_{c}\left(\bar{L}_{c}^{-1} p, \bar{L}_{c}^{-1} p\right)\right\|_{H^{-n}(d \theta)}$ are bounded on $[0, T)$.

Then we can apply [8, Theorem 10.5.5] to conclude that $[0, T)$ is not the maximal interval of existence. Since this holds for every $T$, the geodesic must exist on $[0, \infty)$.

Assume now that $T>0$ is fixed. We will pass freely between the momentum and the velocity via $u(t)=\bar{L}_{c(t)}^{-1} p(t)$. Since $c(t)$ is a geodesic, we have

$$
\operatorname{dist}^{G}\left(c_{0}, c(t)\right) \leqslant \sqrt{G_{c(0)}(u(0), u(0))} T
$$

and

$$
G_{c(t)}(u(t), u(t))=G_{c(0)}(u(0), u(0)) .
$$

In particular, the geodesic remains in a metric ball around $c_{0}$. It follows from Corollary 4.11 that there exists a $C>0$ with $\left|c^{\prime}(t, \theta)\right| \geqslant C$ for $(t, \theta) \in[0$, $T) \times S^{1}$. Since the set $\left\{c:\left|c^{\prime}(\theta)\right| \geqslant C\right\}$ is $H^{2}$-closed (and hence also $H^{n}$-closed) in $\operatorname{Imm}^{n}\left(S^{1}, \mathbb{R}^{2}\right)$, we can conclude that condition (A) is satisfied. 
The first part of condition (B) follows easily from

$$
\begin{aligned}
\left\|\bar{L}_{c}^{-1} p\right\|_{H^{n}(d \theta)}^{2} & =\|u\|_{H^{n}(d \theta)}^{2} \lesssim_{c_{0}, T}\|u\|_{H^{n}(d s)}^{2} \\
& \leqslant \max \left(a_{0}^{-1}, a_{n}^{-1}\right) G_{c}(u, u)=\max \left(a_{0}^{-1}, a_{n}^{-1}\right) G_{c(0)}(u(0), u(0)),
\end{aligned}
$$

using Lemma 5.1 and that the velocity is constant along a geodesic.

It remains to show that $\left\|H_{c}(u, u)\right\|_{H^{-n}(d \theta)}$ remains bounded along $c(t)$. To estimate this norm, pick $m \in H^{n}(d \theta)$, and consider the pairing

$$
\begin{array}{r}
\left\langle H_{c}(u, u), m\right\rangle_{H^{-n} \times H^{n}}=D_{c, m} G_{c}(u, u)=\int_{S^{1}} \sum_{k=0}^{n} a_{k}\left\langle D_{s}^{k} u, D_{s}^{k} u\right\rangle\left\langle D_{s} m, v\right\rangle d s \\
-2 \sum_{k=1}^{n} \sum_{j=1}^{k} a_{k}\left\langle D_{s}^{k} u, D_{s}^{k-j}\left(\left\langle D_{s} m, v\right\rangle D_{s}^{j} u\right)\right\rangle \mathrm{d} s .
\end{array}
$$

Using Poincaré inequalities, Lemma 5.1, and that $\ell_{c}$ is bounded along $c(t)$, we can estimate the first term,

$$
\begin{aligned}
\left|\int_{S^{1}} \sum_{k=0}^{n} a_{k}\left\langle D_{s}^{k} u, D_{s}^{k} u\right\rangle\left\langle D_{s} m, v\right\rangle \mathrm{d} s\right| & \leqslant\left\|D_{s} m\right\|_{L^{\infty}} G_{c}(u, u) \\
& \lesssim_{c_{0}, T}\|m\|_{H^{n}(d s)} \lesssim_{c_{0}, T}\|m\|_{H^{n}(d \theta)} .
\end{aligned}
$$

For the second term, we additionally need Corollary 5.2. For each $1 \leqslant k \leqslant n$ and $1 \leqslant j \leqslant k$, we have

$$
\begin{aligned}
& \left|\int_{S^{1}}\left\langle D_{s}^{k} u, D_{s}^{k-j}\left(\left\langle D_{s} m, v\right\rangle D_{s}^{j} u\right)\right\rangle \mathrm{d} s\right| \leqslant\left\|D_{s}^{k} u\right\|_{L^{2}(d s)}\left\|D_{s}^{k-j}\left(\left\langle D_{s} m, v\right\rangle D_{s}^{j} u\right)\right\|_{L^{2}(d s)} \\
& \quad \leqslant\|u\|_{H^{k}(d s)}\left\|\left\langle D_{s} m, v\right\rangle D_{s}^{j} u\right\|_{H^{k-j}(d s)} \\
& \quad \lesssim_{c_{0}, T}\|u\|_{H^{k}(d s)}\left\|D_{s} m\right\|_{H^{k-j}(d s)}\|v\|_{H^{k-j}(d s)}\left\|D_{s}^{j} u\right\|_{H^{k-j}(d s)} \\
& \quad \lesssim_{c_{0}, T}\|u\|_{H^{n}(d s)}^{2}\|c\|_{H^{n}(d s)}\|m\|_{H^{n}(d s)} .
\end{aligned}
$$

We know that $\|u\|_{H^{n}(d s)}^{2}$ is bounded along $c(t)$, and using Lemma 5.3 we see that $\|c\|_{H^{n}(d s)}$ is bounded as well. Hence we obtain

$$
\left|\left\langle H_{c}(u, u), m\right\rangle_{H^{-n} \times H^{n}}\right| \lesssim_{c_{0}, T}\|m\|_{H^{n}(d \theta)},
$$

which implies that

$$
\left\|H_{c}(u, u)\right\|_{H^{-n}(d \theta)}{c_{0}, T}_{1} 1
$$

that is, $\left\|H_{c}(u, u)\right\|_{H^{-n}(d \theta)}$ is bounded along the geodesic. 
REMARK 5.7. If $G$ is a Sobolev-type metric of order $n \geqslant 2$ with $a_{0}=0, a_{1}=0$, then $G$ is a Riemannian metric on the space $\operatorname{Imm}\left(S^{1}, \mathbb{R}^{2}\right) /$ Tra of plane curves modulo translations. We will show that for these metrics it is possible to blow up circles to infinity along geodesics in finite time, making them geodesically incomplete. Thus a nonvanishing zero or first-order term is necessary for geodesic completeness.

The one-dimensional submanifold consisting of concentric circles, that are parameterized by constant speed, is a geodesic with respect to the metric, because Sobolev-type metrics are invariant under the motion group. Let $c(t$, $\theta)=r(t)(\cos \theta, \sin \theta)$. Then $c_{t}(t, \theta)=r_{t}(t)(\cos \theta, \sin \theta)$ and $\left|c^{\prime}(t, \theta)\right|=r(t)$. Thus

$$
G_{c}\left(c_{t}, c_{t}\right)=2 \pi \sum_{j=2}^{n} a_{j} r(t)^{1-2 j} r_{t}(t)^{2}
$$

and the length of the curve is

$$
\operatorname{Len}^{G}(c)=\int_{0}^{1} \sqrt{2 \pi \sum_{j=2}^{n} a_{j} r(t)^{1-2 j} r_{t}(t)^{2}} d t=\sqrt{2 \pi} \int_{r(0)}^{r(1)} \sqrt{\sum_{j=2}^{n} a_{j} \sigma^{1-2 j}} \mathrm{~d} \sigma .
$$

Since the integral converges for $r(1) \rightarrow \infty$, it follows that the path consisting of growing circles can reach infinity with finite length.

\section{References}

[1] R. A. Adams, Sobolev Spaces, 2nd edn (Academic Press, 2003).

[2] M. Bauer, M. Bruveris and P. W. Michor, 'Overview of the geometries of shape spaces and diffeomorphism groups', J. Math. Imaging Vision (2014), doi: 10.1007/s10851-013-0490-z.

[3] M. Bauer, M. Bruveris and P. W. Michor, ' $R$-transforms for Sobolev $H^{2}$-metrics on spaces of plane curves', Geom. Imaging Comput. 1 (1) (2014), 1-56.

[4] M. Bauer, P. Harms and P. W. Michor, 'Sobolev metrics on shape space of surfaces', J. Geom. Mech. 3 (4) (2011), 389-438.

[5] M. Bauer, P. Harms and P. W. Michor, 'Sobolev metrics on shape space, II: Weighted Sobolev metrics and almost local metrics', J. Geom. Mech. 4 (4) (2012), 365-383.

[6] M. Bruveris and F.-X. Vialard, 'On completeness of groups of diffeomorphisms', (2014), preprint: arXiv:1403.2089.

[7] G. Charpiat, P. Maurel, J.-P. Pons, R. Keriven and O. Faugeras, 'Generalized gradients: Priors on minimization flows', Int. J. Comput. Vis. 73 (3) (2007), 325-344.

[8] J. Dieudonné, Foundations of Modern Analysis, Enlarged and corrected printing, Pure and Applied Mathematics, vol. 10-I (Academic Press, New York, 1969).

[9] D. G. Ebin and J. Marsden, 'Groups of diffeomorphisms and the motion of an incompressible fluid', Ann. of Math. (2) 92 (1970), 102-163.

[10] F. Faà di Bruno, 'Note sur une nouvelle formule du calcul difféntielle', Quart. J. Math. 1 (1855), 359-360. 
[11] H. Inci, T. Kappeler and P. Topalov, On the Regularity of the Composition of Diffeomorphisms, Memoirs of the American Mathematical Society, vol. 226 (American Mathematical Society, 2013).

[12] G. S. Jones, 'Fundamental inequalities for discrete and discontinuous functional equations', J. Soc. Ind. Appl. Maths 12 (1964), 43-57.

[13] A. Kriegl and P. W. Michor, The convenient setting of global analysis, Mathematical Surveys and Monographs, vol. 53 (American Mathematical Society, Providence, RI, 1997).

[14] S. Kurtek, A. Srivastava, E. Klassen and Z. Ding, 'Statistical modeling of curves using shapes and related features', J. Am. Stat. Assoc. 107 (499) (2012), 1152-1165.

[15] A. Mennucci, A. Yezzi and G. Sundaramoorthi, 'Properties of Sobolev-type metrics in the space of curves', Interfaces Free Bound. 10 (4) (2008), 423-445.

[16] M. Micheli, P. W. Michor and D. Mumford, 'Sobolev metrics on diffeomorphism groups and the derived geometry of spaces of submanifolds', Izv. Math. 77 (3) (2013), 541-570.

[17] P. W. Michor and D. Mumford, 'Riemannian geometries on spaces of plane curves', J. Eur. Math. Soc. (JEMS) 8 (2006), 1-48.

[18] P. W. Michor and D. Mumford, 'An overview of the Riemannian metrics on spaces of curves using the Hamiltonian approach', Appl. Comput. Harmon. Anal. 23 (1) (2007), 74-113.

[19] D. Mumford and P. W. Michor, 'On Euler's equation and 'EPDiff', J. Geom. Mech. 5 (3) (2013), 319-344.

[20] L. Nirenberg, 'On elliptic partial differential equations', Ann. Scuola Norm. Sup. Pisa (3) 13 (1959), 115-162.

[21] F. W. J. Olver, D. W. Lozier, R. F. Boisvert and C. W. Clark (Eds.), NIST Handbook of Mathematical Functions (US Department of Commerce National Institute of Standards and Technology, Washington, DC, 2010).

[22] B. G. Pachpatte, Inequalities for differential and integral equations, Mathematics in Science and Engineering, vol. 197 (Academic Press Inc., San Diego, CA, 1998).

[23] G. Sundaramoorthi, A. Mennucci, S. Soatto and A. Yezzi, 'A new geometric metric in the space of curves, and applications to tracking deforming objects by prediction and filtering', SIAM J. Imaging Sci. 4 (1) (2011), 109-145.

[24] G. Sundaramoorthi, A. Yezzi and A. C. Mennucci, 'Sobolev active contours', Int. J. Comput. Vis. 73 (3) (2007), 345-366.

[25] A. Trouvé and L. Younes, 'Local geometry of deformable templates', SIAM J. Math. Anal. 37 (1) (2005), 17-59. electronic. 